\section{Prospects in the management of patients with follicular lymphoma beyond first-line therapy}

\author{
David Qualls ${ }^{1}$ and Gilles Salles ${ }^{1,2}$
}

${ }^{1}$ Lymphoma Service, Department of Medicine, Memorial Sloan Kettering Cancer Center and ${ }^{2}$ Weill Cornell Medicine, New York, NY, USA

\section{ABSTRACT}

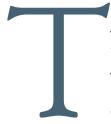
he management of patients with relapsed or refractory follicular lymphoma has evolved markedly in the last decade, with the availability of new classes of agents (phosphoinositide 3-kinase inhibitors, immunomodulators, epigenetic therapies, and chimeric antigen receptor $\mathrm{T}$ cells) supplementing the multiple approaches already available (cytotoxic agents, anti-CD20 antibodies, radiation therapy, radioimmunotherapy, and autologous and allogeneic transplants). The diversity of clinical scenarios, the flood of data derived from phase II studies, and the lack of randomized studies comparing treatment strategies preclude firm recommendations and require personalized decisions. Patients with early progression require specific attention given the risk of histological transformation and their lower response to standard therapies. In sequencing therapies, one must consider prior treatment regimens and the potential need for future lines of therapy. Careful evaluation of risks and expected benefits of available options, which vary depending on location and socioeconomics, should be undertaken, and should incorporate the patient's goals. Preserving quality of life for these patients is essential, given the likelihood of years to decades of survival and the possibility of multiple lines of therapy. The current landscape is likely to continue evolving rapidly with other effective agents emerging (notably bispecific antibodies and other targeted therapies), and multiple combinations being evaluated. It is hoped that new treatments under development will achieve longer progression-free intervals and minimize toxicity. A better understanding of disease biology and the mechanisms of these different agents should provide further insights to select the optimal therapy at each stage of disease.

\section{Introduction}

While the median overall survival (OS) of patients with follicular lymphoma (FL) was under 10 years over two decades ago, the vast majority $(\sim 0 \%)$ of patients diagnosed today are likely to be alive 10 years after their initial diagnosis, and their expected median OS may exceed 20 years. ${ }^{1}$ This remarkable progress reflects the improved efficacy of first-line therapeutic interventions with the introduction of anti-CD20 antibodies, and our ability to deliver active therapies in subsequent lines for those patients experiencing disease progression. Several important challenges should be considered regarding the treatment of patients with disease progression after first-line therapy, who are still generally considered to have an incurable disease after the lymphoma recurrence.

The first challenge is the possibility of histological transformation - the leading cause of death of patients with $\mathrm{FL}^{2}$ Transformation can occur any time during the disease course, although some data suggest an increased risk in the early years after diagnosis which decreases thereafter. ${ }^{2-4}$ This feared event should always be anticipated, and a new tumor biopsy performed whenever lymphoma progresses or does not respond to therapy. The use of positron emission tomography/computed tomography to guide the site of biopsy in the nodal area with the highest glucose uptake is recommended. The detailed management of transformed FL has been recently reviewed ${ }^{5}$ and is beyond the scope of this paper.

The second consideration is the optimal sequencing of available therapies. ${ }^{6}$
Haematologica 2022

Volume 107(1):19-34

\section{Correspondence:}

GILLES SALLES

sallesg@mskcc.org

Received: August 31, 2021.

Accepted: October 5, 2021.

https://doi.org/10.3324/haematol.2021.278717

(C)2022 Ferrata Storti Foundation

Material published in Haematologica is covered by copyright. All rights are reserved to the Ferrata Storti Foundation. Use of published material is allowed under the following terms and conditions:

https://creativecommons.org/licenses/by-nc/4.0/legalcode. Copies of published material are allowed for personal or internal use. Sharing published material for non-commercial purposes is subject to the following conditions:

https://creativecommons.org/licenses/by-nc/4.0/legalcode, sect. 3. Reproducing and sharing published material for commercial purposes is not allowed without permission in writing from the publisher. 
While some clinical trials were conducted in specific populations of patients, such as those with disease refractory to rituximab or to rituximab and alkylating agents ("double refractory"), there are few randomized clinical trials to guide our choice of a given therapy at a precise time. This is in part due to the broad array of options available after diagnosis, with a variety of mechanisms of action (Figure 1 ), and to the heterogeneity of patients entering clinical trials evaluating new agents in the relapsed/refractory setting. However, not all available drugs or regimens have similar clinical efficacy - both in terms of response rate, and more importantly, response duration.
A third key element is the patients' quality of life, including short-term treatment-related side effects and convenience of therapeutic administration, as well as long-term and cumulative toxicities, which may result in cardiac, hematopoietic, infectious, or neurological comorbidities, or increase the risk of potentially fatal secondary malignancies. Attractive oral therapies have emerged, although they have their own toxicity profiles, and are often administered for indefinite periods, presenting unique challenges. Overall, these different elements should be discussed with every patient, with transparency regarding the benefits and risks of each strategy, keep-

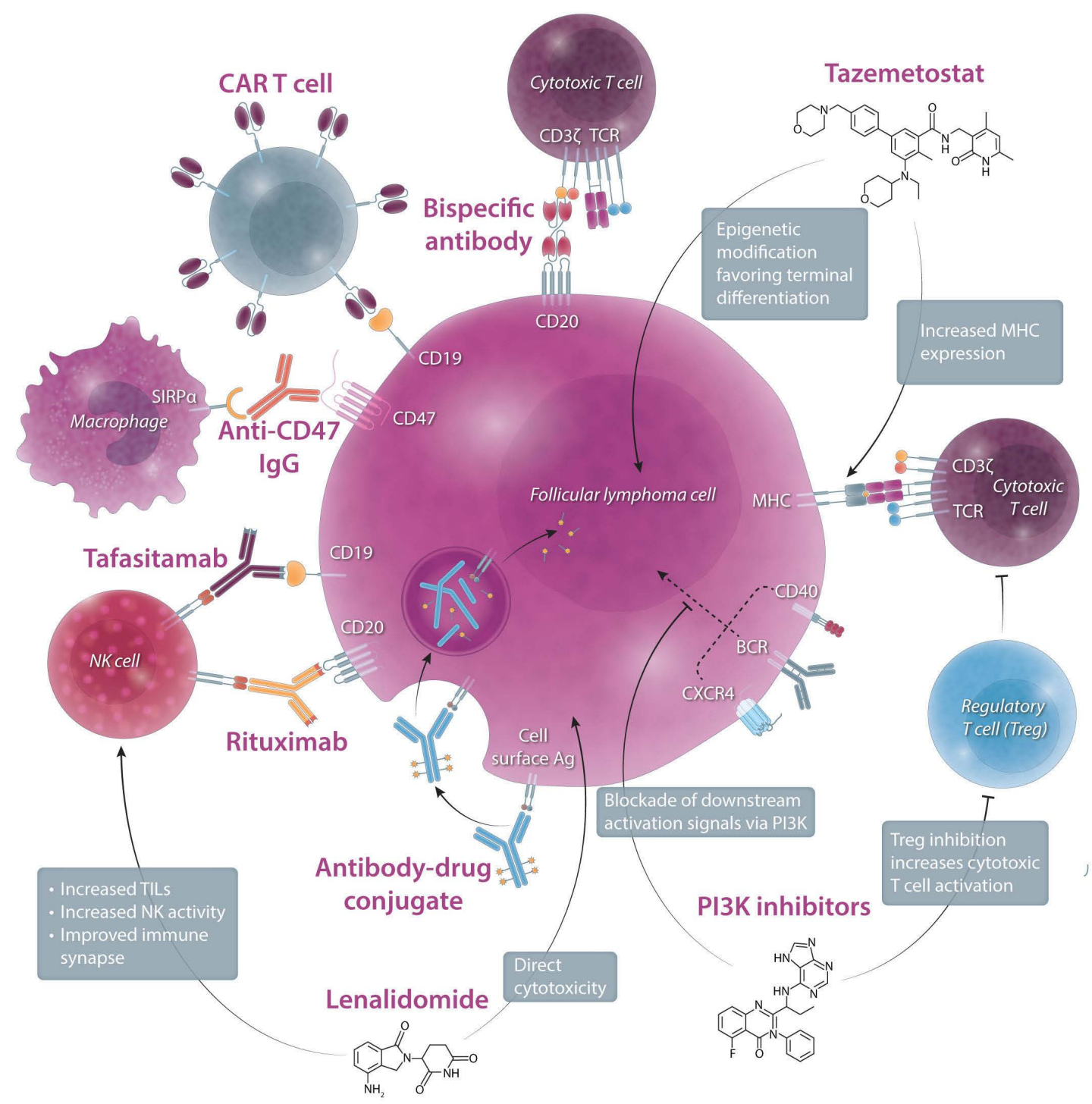

Figure 1. Mechanisms of action and opportunities for synergy in follicular lymphoma-directed therapies. From top left, clockwise: Autologous chimeric antigen receptor T cells are engineered to target the CD19 epitope on follicular lymphoma (FL) cells, with co-stimulatory domains activating T-cell anti-tumor responses. Bispecific antibodies bind both CD2O on the lymphoma cells and CD3 on the surface of cytotoxic T cells, activating anti-tumor cytotoxicity. Tazemetostat inhibits EZH2mediated suppression of differentiation genes in FL cells, and inhibits suppression of MHC expression, allowing for greater immune recognition of lymphoma cells. PI3K inhibitors block key molecular signal pathways for the growth and survival of lymphoma cells, and also inhibit T regulatory cell function, which may facilitate immune activation against FL cells. Lenalidomide functions via cereblon-mediated ubiquitination and degradation of transcriptional factors, which is directly cytotoxic to lymphoma cells, and potentiates the immune synapse, improving T-cell- and NK-cell-mediated recognition and killing of lymphoma cells. Lenalidomide is synergistic with monoclonal antibodies (rituximab and tafasitamab), which together promote antibody-dependent cellular phagocytosis and antibody-dependent cellular cytotoxicity. Antibody-drug conjugates bind to the cell expressing the target antigen, are internalized, and deliver their cytotoxic payload directly in the cytoplasm. CD47 on FL interacts with SIRP $\alpha$ on macrophages to inhibit phagocytosis of cancer cells. Anti-CD47 antibodies block this checkpoint interaction, promoting macrophage phagocytosis. CAR: chimeric antibody receptor; TCR: T-cell receptor; IgG: immunoglobulin G; MHC: major histocompatibility complex; BCR: B-cell receptor; Ag: antigen; PI3K: phosphoinositide 3-kinase; TILs: tumor-inflitrating lymphocytes; NK: natural killer. 
ing in mind our goals (prolongation of OS while preserving quality of life) as well as patients' own priorities, which might differ according to their age and their personal history. ${ }^{8}$

\section{Prognostic factors in patients with relapsed or refractory disease}

While many discoveries have advanced our understanding of the biology of FL, from patterns of gene mutations to the immune microenvironment, efforts to establish biological parameters that predict individual patients' outcomes have yielded few reliable indicators to date. The extreme clonal heterogeneity and diversification of FL over time point towards the frequent emergence of divergent clones originating from a common progenitor cell, rather than a selective, directional evolutionary process. One suspects that rapidly resistant and evolutive disease may accumulate alterations accounting for chemoresistance, but such alterations have yet to be identified, aside from genetic changes occurring at the time of histological transformation. ${ }^{9,10}$

The Follicular Lymphoma International Prognostic Index (FLIPI) has been shown to be predictive of the outcome of patients at the time of first lymphoma progression, and one usually assumes that clinical or biological features identified as prognostic factors at diagnosis may apply at the time of progression, although this has not been properly examined in the last decade in large series. ${ }^{11}$ The clinical impact of biological characteristics (genomic gain or loss, point mutations, immune and microenvironment changes) present at the time of disease progression has not been evaluated extensively, and represents an area for further investigation, especially given the growing accessibility of therapies with distinct mechanisms of action.

In recent years, attention has been drawn to the poor outcome of patients experiencing early disease progression after their initial treatment. This has been well documented after immunochemotherapy, with worse outcomes associated with disease progression within 12 or 24 months of initial treatment, described in shorthand as EFS12 or EFS24/POD24 disease, respectively. ${ }^{12,13}$ These findings have been reproduced in patients treated with rituximab as a single agent or in combination with lenalidomide, as well as in those having received radiation therapy for localized disease. ${ }^{14,15}$ Patients under observation for whom an event such as treatment initiation or clinical progression within 12 months occurs were similarly found to have a shorter survival. ${ }^{13}$ These findings have led some to advocate for more intensive therapy (such as autologous stem cell transplant - ASCT) in patients with POD24 disease. ${ }^{16}$ However, most of these retrospective studies did not account for cases with histological transformation, which are associated with poor outcomes. ${ }^{17}$ Notably, recent data indicate that a high proportion of patients with early progression present with histological transformation, with numbers ranging from 20 to $40 \%$ in patients having received CHOP (cyclophosphamide, adriamycin, vincristine, prednisone) or CVP (cyclophosphamide, vincristine, prednisone) regimens (containing an anti-CD20 antibody) and from 20 to $75 \%$ of those having received bendamustine as a cytotoxic backbone. ${ }^{3,17}$
The precise evaluation of treatment efficacy in patients with POD24 is hampered by the lack of pre-treatment biopsy requirements in some studies, as well as subtle variations in the definition of POD24 disease in others. However, some new agents or combinations remain active in this setting, suggesting that new approaches might potentially circumvent this adverse disease feature.

\section{Old tools remain useful in the relapsed and refractory settings}

\section{Repeating chemoimmunotherapy: still a standard of care}

While promising targeted agents and immunotherapies are now available, treatments incorporating chemotherapy and anti-CD20 monoclonal antibodies remain a mainstay of treatment for the management of relapsed/refractory FL. The choice of agents depends on patients' initial therapy, with preference for a non-cross-resistant option.

We have the most robust clinical data to guide decisionmaking in patients who received first-line therapy with rituximab plus CHOP (R-CHOP) or rituximab plus CVP (R-CVP), wherein bendamustine-based therapy is most frequently utilized. The StiL study demonstrated superior progression-free survival (PFS) with rituximab plus bendamustine (B-R) over rituximab plus fludarabine in a randomized study of relapsed/refractory indolent nonHodgkin lymphoma, with a PFS of 34.2 months for B-R versus 11.7 months for rituximab plus fludarabine, and an OS benefit with B-R. ${ }^{18}$ In this study, $50 \%$ of patients had $\mathrm{FL}$, with the majority having been previously treated with first-line CHOP-based therapy. The GADOLIN phase III clinical trial established the efficacy of obinutuzumab plus bendamustine with obinutuzumab maintenance as another second-line option in patients with rituximabrefractory $\mathrm{FL}$, defined as primary resistance or relapse within 6 months of rituximab-containing therapy. Compared to bendamustine alone, obinutuzumab plus bendamustine and obinutuzumab maintenance demonstrated PFS and OS benefits in the overall cohort of nonHodgkin lymphoma patients and in the subset with FL. ${ }^{19,20}$ Of note, bendamustine regimens were associated with prolonged T-cell depletion in other studies, and precautions, including anti-microbial prophylaxis, should be considered. $^{21}$

Important gaps remain in our knowledge regarding second-line therapy. In rituximab-sensitive individuals, rituximab and obinutuzumab have not been compared directly. While GADOLIN investigated rituximab-refractory disease, the lack of rituximab in the control arm also raises questions about whether obinutuzumab is truly superior to rituximab in disease defined as rituximab-refractory.

Treatment after first-line bendamustine-based therapy is poorly established as there are no dedicated trials evaluating this population. Large long-term outcome studies in FL have included very few patients receiving first-line $\mathrm{B}-\mathrm{R}$, likely representing its more recent adoption. ${ }^{22,23}$ Convention has been to use non-cross-resistant chemotherapy, such as rituximab or obinutuzumab (R/O) with $\mathrm{CHOP}$ or R/O-CVP. One small retrospective study of bendamustine-based re-challenge in patients with chronic lymphocytic leukemia or lymphoma showed a response rate of $78 \%$ with $B-R$ in lymphoma, with grade 
3-4 hematologic toxicity following 35\% of treatment administrations. ${ }^{24}$ Given the availability of alternative agents, along with concerns regarding cumulative hematologic toxicity and risk for secondary malignancies, we continue to favor $\mathrm{R} / \mathrm{O}-\mathrm{CHOP}$ or R/O-CVP over B-R retreatment if chemotherapy is needed. However, this remains a major information gap in FL therapy. Given the increased use of first-line $B-R$, more studies in this area are needed.

As for B-R, few data are available regarding second-line therapy after the rituximab-lenalidomide (R2) combination has been used as first-line treatment. ${ }^{25} \mathrm{~A}$ recent retrospective study evaluated outcomes in patients with relapsed or progressive FL who received R2 as first-line therapy. ${ }^{26}$ The overall response rate was $78 \%$ and median PFS was 38 months after salvage therapy, and the use of chemoimmunotherapy (B-R or R-CHOP) was associated with a significantly longer PFS compared to that achieved with biological agents, supporting the use of immunochemotherapy regimens in this second-line context.

Other chemoimmunotherapy regimens have demonstrated activity in relapsed/refractory FL, although they are less commonly implemented. These include fludarabinebased combinations (with cyclophosphamide, mitoxantrone, or both), rarely utilized because of their substantial hematologic toxicities, immunosuppression, and risk of secondary neoplasias. ${ }^{27}$ Cytarabine-containing regimens (such as R-DHAOX [rituximab, dexamethasone, cytarabine, oxaliplatin] and R-DHAP [rituximab, dexamethasone, cytarabine, cisplatin]) showed encouraging results in a retrospective study in patients with early relapsed or refractory disease, often as a path towards high-dose therapy and ASCT. ${ }^{28}$ The use of chlorambucil (and less commonly cyclophosphamide) in combination with rituximab represents an alternative in comorbid or frail patients. ${ }^{2,30}$

\section{The role of anti-CD20 maintenance after second line chemoimmunotherapy}

Following successful second-line treatment with response, maintenance therapy with rituximab or obinutuzumab is often considered. Two large randomized clinical trials by the European Organisation for Research and Treatment of Cancer and the German Low Grade Lymphoma Study Group demonstrated PFS benefit with maintenance rituximab after second-line chemoimmunotherapy ${ }^{31,32} \mathrm{~A}$ subsequent meta-analysis of individual patients' data from randomized clinical trials evaluating maintenance rituximab found an OS benefit with the use of maintenance rituximab after second-line induction therapy ${ }^{33}$ However, many patients in these trials had not received rituximab during their frontline management (and specifically no anti-CD20 maintenance), and since these trials were completed a number of new treatment options for FL have been approved, which may affect any survival benefit with maintenance rituximab.

The decision of whether to pursue maintenance rituximab or obinutuzumab should be based on prior therapy and the most recent progression-free interval might be considered. For patients with rituximab-refractory disease, obinutuzumab-based treatment as per GADOLIN would be preferred, whereas in rituximab-sensitive disease continued rituximab is reasonable. In these studies, maintenance treatment was generally well tolerated, although with an increase in grade 3-4 infections.

\section{Should a therapeutic response in the relapsed or refractory setting be consolidated with transplantation?}

Consolidation high-dose chemotherapy with stem cell rescue can be considered in the relapsed/refractory setting, particularly in those with high-risk disease. An early randomized study of relapsed/refractory FL noted PFS and OS benefits with high-dose chemotherapy and ASCT after initial response to chemotherapy (predominantly (HOP), as opposed to additional chemotherapy alone, although it is difficult to extrapolate these findings which preceded the routine use of rituximab. ${ }^{34}$ Other studies have demonstrated durable responses following consolidative autologous transplant (with a median PFS exceeding 5 years in one large study), with a suggestion of greater benefit in patients with early progression. ${ }^{35-38}$ It should be considered that better disease control in patients presenting with histological transformation may have contributed to some of the benefit seen with ASCT in early progression, given the high rates of such transformation in POD24. One study stratifying outcomes by histological transformation showed a clear OS benefit with ASCT in those with transformation, while in those without, ASCT was not associated with improved survival. ${ }^{39}$ Overall, the sustained PFS benefit achieved with ASCT for patients with FL should be balanced with the immediate and long-term toxicities of the procedure, particularly the risk of secondary malignancies ${ }^{40}$ Indications for ASCT also need to be interpreted in the context of alternative treatment options, such as antibody-based therapies and, more recently, chimeric antigen receptor (CAR) T-cell therapy.

Allogeneic transplant with reduced intensity conditioning also remains a viable option with prolonged periods of disease-free survival. However, this must be balanced with the relatively high treatment-related morbidity and mortality, with other options including chemoimmunotherapy, CAR T cells, and other immune-based therapies offering more favorable toxicity profiles. We consider this option for patients with multiply refractory FL, particularly younger, otherwise healthy patients with good performance status and adequate candidate donors.

\section{Radiation and radioimmunotherapy: potent tools for local and systemic treatment}

FL is a highly radiosensitive disease, and both external radiation and radio-immunotherapies are effective tools in the management of relapsed or refractory FL.

While extended-field radiation with high doses might expose patients to long-term sequelae, focal radiotherapy to symptomatic lymph nodes represents an efficacious approach which is easy to administer. The optimal dose of radiation in this scenario is an area of active discussion. A recent study comparing standard 24 Gy dosing with a $2 \times 2$ (4 Gy) regimen demonstrated significantly superior local control with higher dose therapy (5-year progression-free rates of 89.9 vs. $70.4 \%$, respectively), although no difference in OS was observed, and more acute toxicity was seen in the $24 \mathrm{~Gy}$ arm. ${ }^{41}$ In our hands, a 2-year local control rate of $70 \%$ has been achieved with 2 x 2 Gy in patients with recurrent disease, and this option appears remarkably versatile in patients with lesions $<6 \mathrm{~cm}$ (Imber B et al., Blood Adv, in press). Based on the lack of curative intent in the relapsed/refractory setting, reduced 
toxicity, and the opportunity for re-treatment, we favor the $2 \times 2$ Gy approach for local treatment of symptomatic relapsed/refractory FL.

For systemic treatment, radioimmunotherapy was developed in the last two decades with ${ }^{131} \mathrm{I}$ coupled to tositumomab and ${ }^{90}$ Y-ibritumomab tiuxetan, which produced high rates of response. ${ }^{42}$ Various logistical and economic considerations account for the lack of widespread use of these agents, and only ibritumomab remains available in North America and Europe. Furthermore, since both agents target the CD20 antigen, their efficacy in patients recently exposed to rituximab is possibly hindered by competition with rituximab for target antigens. To circumvent this, radioimmunoconjugates targeting different cell surface antigens have been developed. Encouraging preliminary results were noted with ${ }^{17} \mathrm{Lu}-$ lilotomab satetraxetan, a CD37-directed antibody, in patients with refractory FL, and a registration phase II study is currently underway (NCT01796171). ${ }^{43}$

\section{Targeted therapies: exploiting tumor cell vulnerabilities}

\section{Benefits and limits of phosphoinositide 3-kinase inhibitors}

FL and other lymphomas exhibit constitutive overactivation of the phosphoinositide 3-kinase (PI3K)-AKT-mecha- nistic target of rapamycin (mTOR) pathway, which has important roles in proliferation, growth, and survival of both normal and cancer cells. ${ }^{44}$ Signaling through the B-cell receptor and other cell-surface receptors, which might be critical for lymphoma survival, also depends on this pathway. As a result, PI3K is now a target of significant interest in treating FL, with a number of targeted inhibitors demonstrating activity in multiply refractory disease, and four agents approved in the USA (Table 1). ${ }^{45-49}$ Approved PI3K inhibitors have all been evaluated in single-arm phase II studies, limiting comparison between PI3K inhibitors or with other treatment modalities (Table 1). Response rates were between $42 \%$ and $66 \%$ and the median PFS was between 10 and 13 months for the four agents, which were all evaluated in FL that had relapsed after or had been refractory to at least two prior lines of treatment. Differences in the efficacy and toxicity of these inhibitors are largely related to the specific subunits targeted by the therapy. ${ }^{44}$ Class 1 PI3K is composed of a heterodimer containing a 110 subunit $(\alpha, \beta, \gamma$ or $\delta)$ and a regulatory subunit. The $\gamma$ and $\delta$ subunits are more specifically expressed in leukocytes, whereas the $\alpha$ and $\beta$ subunits are widely expressed in normal tissue, although all have been implicated in lymphomagenesis.

Idelalisib, the first approved PI3K inhibitor, is $\delta$-specific, and was shown to generate high response rates in FL refractory to at least two prior lines of treatment including anti-CD20 and alkylator therapy. ${ }^{45,46}$ Complete

Table 1. Selected trials and outcomes with targeted therapies in relapsed or refractory follicular lymphoma.

\begin{tabular}{|c|c|c|c|c|c|c|c|c|}
\hline PI3K inhibitors (approved) & Phase & $\begin{array}{l}\text { Prior lines } \\
\text { of therapy }\end{array}$ & $\begin{array}{l}\text { N. of patients } \\
\text { with FL }\end{array}$ & ORR & CRR & $\begin{array}{l}\text { PFS, median } \\
\text { (months) }\end{array}$ & $\begin{array}{l}\text { DoR, median } \\
\text { (months) }\end{array}$ & OS \\
\hline Idelalisib $^{46}$ & II & $\geq 2$ & 72 & 66 & 14 & 11 & 11 & $2 y: 70 \%$ \\
\hline Duvelisib $^{47}$ & II & $\geq 2$ & 83 & 42 & 1 & 10 & 10 & $2 y: 60 \%$ \\
\hline Umbralisib $^{48}$ & II & $\geq 2$ & 117 & 45 & 5 & 11 & 11 & N/A \\
\hline Copanlisib ${ }^{49}$ & II & $\geq 2$ & 104 & 59 & 20 & 13 & 14 & $2 y: 69 \%$ \\
\hline $\begin{array}{l}\text { Copanlisib + rituximab }{ }^{53} \\
\text { Rituximab control arm }\end{array}$ & $\begin{array}{l}\text { III } \\
\text { III }\end{array}$ & $\begin{array}{l}\geq I \\
\geq 1\end{array}$ & $\begin{array}{c}184 \\
91\end{array}$ & $\begin{array}{l}85 \\
54\end{array}$ & $\begin{array}{l}37 \\
21\end{array}$ & $\begin{array}{l}22 \\
19\end{array}$ & $\begin{array}{l}20.4^{*} \\
17.3^{*}\end{array}$ & $\begin{array}{l}3 y: 83 \% \\
\text { 3y: } 81 \%\end{array}$ \\
\hline \multicolumn{9}{|l|}{ PI3K inhilbitors (ongoing studies) } \\
\hline Parsaclisib (daily dosing) (1) $^{21}$ & II & $\geq 2$ & 74 & 72 & 13.5 & 15.8 & NR & N/A \\
\hline Parsaclisib (weekly dosing) (1) $^{51}$ & II & $\geq 2$ & 22 & 64 & 13.6 & N/A & $\mathrm{N} / \mathrm{A}$ & $\mathrm{N} / \mathrm{A}$ \\
\hline $\begin{array}{l}\text { Zandelisib + rituximab } \\
\text { Zandelisib }\end{array}$ & $\begin{array}{l}\mathrm{Ib} \\
\mathrm{Ib}\end{array}$ & $\begin{array}{l}\geq 1 \\
\geq 1\end{array}$ & $\begin{array}{c}9 \\
39\end{array}$ & $\begin{array}{l}78 \\
79\end{array}$ & $\begin{array}{l}\mathrm{N} / \mathrm{A} \\
\mathrm{N} / \mathrm{A}\end{array}$ & $\begin{array}{l}\text { N/A } \\
\text { N/A }\end{array}$ & $\begin{array}{l}\mathrm{N} / \mathrm{A} \\
\mathrm{N} / \mathrm{A}\end{array}$ & $\begin{array}{l}\text { N/A } \\
\text { N/A }\end{array}$ \\
\hline \multicolumn{9}{|l|}{ BTK inhilbitors } \\
\hline Ibrutinib $^{54}$ & II & $\geq 2$ & 110 & 21 & 11 & 4.6 & 19.4 & ly $78 \%$ \\
\hline Acalabrutinib $^{56}$ & $\mathrm{Ib}$ & $\geq 1$ & 12 & 33 & 8 & N/A & NR & N/A \\
\hline Acalabrutinib + rituximab $b^{56}$ & $\mathrm{Ib}$ & $\geq 1$ & 13 & 39 & 8 & N/A & NR & $\mathrm{N} / \mathrm{A}$ \\
\hline Zanubrutinib + obinutuzumab ${ }^{57}$ & $\mathrm{Ib}$ & $\geq 1$ & 36 & 72 & 39 & 25 & NR & N/A \\
\hline \multicolumn{9}{|l|}{ mTOR inhibitors } \\
\hline Everolimus $^{58}$ & II & $\geq 1$ & 23 & 61 & $\mathrm{~N} / \mathrm{A}$ & $7.2^{*}$ & $\mathrm{~N} / \mathrm{A}$ & median: 29.4 mo \\
\hline Temsirolimus $^{59}$ & II & $\geq 1$ & 39 & 54 & 26 & 12.7 & 13.3 & 3y: $73 \%$ \\
\hline \multicolumn{9}{|l|}{ BCL2 inhibitors } \\
\hline Venetoclax $^{60}$ & I & $\geq 1$ & 29 & 38 & 17 & 11 & 27 & N/A \\
\hline $\begin{array}{l}\text { Venetoclax - BR } \\
\text { BR control arm }\end{array}$ & $\begin{array}{l}\text { II } \\
\text { II }\end{array}$ & $\begin{array}{l}\geq 1 \\
\geq 1\end{array}$ & $\begin{array}{l}51 \\
51\end{array}$ & $\begin{array}{l}84 \\
84\end{array}$ & $\begin{array}{l}75 \\
69\end{array}$ & $\begin{array}{l}\text { N/A } \\
\text { N/A }\end{array}$ & $\begin{array}{l}\text { N/A } \\
\text { N/A }\end{array}$ & $\begin{array}{l}\text { N/A } \\
\text { N/A }\end{array}$ \\
\hline Venetoclax - rituximab ${ }^{61}$ & II & $\geq 1$ & 52 & 35 & 17 & 6.6 & N/A & N/A \\
\hline
\end{tabular}

* Overall population including patients with chronic lymphocytic leukemia/small cell lymphoma, marginal zone lymphoma and follicular lymphoma. Data were collected from published papers and abstracts; cross-trial comparisons are not possible because of different eligibility criteria and other differences. FL: follicular lymphoma; ORR: overall response rate; CRR: complete response rate; PFS: progression-free survival; DoR: duration of response; OS: overall survival; PI3K = phosphoinositide 3-kinase; BTK: Bruton tyrosine kinase; mTOR: mechanistic target of rapamycin; BR: bendamustine and rituximab; NR: not reached; N/A: not available or not reported; y: years; mo: months. 
responses, while rare, appear to be durable, and idelalisib has been shown to have a similar efficacy in patients who experienced early progression after first-line therapy. ${ }^{45,50}$ Copanlisib, a pan-PI3K inhibitor with specificity for the $\alpha$ and $\delta$ isoforms, had similar activity in patients who had received at least two lines of therapy but requires weekly intravenous infusions for 3 or 4 weeks. ${ }^{49}$ Duvelisib is a $\delta$ and $\gamma$-specific PI3K inhibitor and umbralisib is a $\delta$ - and casein kinase-1-specific inhibitor. With the limitation of cross-trial comparisons and a possible learning curve during the development of the different PI3K inhibitors, umbralisib appears to have a better tolerability than that of idelalisib or duvelisib. ${ }^{48}$

There are major limitations regarding PI3K inhibitor monotherapy, including the limited duration of response with a median PFS of approximately 1 year and toxicities with chronic therapy. Class toxicities, including diarrhea/colitis, transaminase elevations, rash, neutropenia, and rarely pneumonitis, along with infectious complications, limit the utility of these agents. Due to the $\alpha$ isoform specificity of copanlisib, toxicities including hypertension and hyperglycemia are unique to copanlisib, and while transient, represent a potential limitation to its use in certain populations.

Other PI3K inhibitors are in development and toxicity profiles may improve with changes in PI3K specificity and dosing regimens. ${ }^{51}$ Currently approved oral PI3K inhibitors are administered on a daily or twice-daily basis throughout treatment. Zandelisib and parsaclisib, $\delta$-specific PI3K inhibitors, are being evaluated using unique dosing schedules intended to mitigate toxicity. Zandelisib was administered on days 1-7 of each 28-day cycle starting in cycle 2 in a phase Ib study. ${ }^{52}$ A phase II study of parsaclisib is evaluating both daily dosing and weekly dosing after an initial 8-week daily dosing period. ${ }^{51}$

The use of combination therapies may offer the greatest opportunity to leverage the efficacy of PI3K inhibitors while addressing the limited duration of responses. A randomized phase III trial, CHRONOS-3, evaluated rituximab with or without copanlisib in relapsed indolent nonHodgkin lymphoma (60\% FL), and demonstrated a PFS benefit from copanlisib with rituximab versus placebo plus rituximab. ${ }^{53}$ The relatively modest prolongation in response duration (20.4 vs. 17.3 months) for patients with FL should be weighed with the constraints of frequent infusions. Further areas of exploration include the combination of other targeted therapies such as BCL2 and mTOR inhibitors. However, caution is still required, as several studies evaluating combinations, including idelalisib plus entospletinib, idelalisib with lenalidomide and rituximab, and dactolisib with abiraterone or everolimus have resulted in unacceptable toxicity profiles. There is also potential for synergy with immunotherapies via $\mathrm{T}$ regulatory cell inhibition, but caution should be employed, as PI3K inhibitor-associated hepatotoxicity and colitis are thought to be immune-mediated and may worsen with immuneactivating therapies such as checkpoint inhibitors.

\section{A challenging path for Bruton tyrosine kinase inhibitors}

FL cells express functional B-cell receptors, and depend on downstream signaling pathways for survival. This provided a rationale to investigate the activity of Bruton tyrosine kinase (BTK) inhibitors in patients. However, in a pivotal single-agent phase II study evaluating 110 patients with double-refractory disease, the overall response rate observed with ibrutinib was only $21 \%$, with a complete response rate of $11 \% .^{54}$ While the duration of response was 19 months, the median PFS was only 5 months (Table 1). These disappointing results might be explained by the biological heterogeneity of FL, the presence of mutations in BTK, or bystander effects of the drug on the microenvironment. ${ }^{55} \mathrm{~A}$ large randomized study (NCT01974440) combining ibrutinib with standard immunochemotherapy regimens has completed accrual, and results are anticipated shortly. Other combinations with ibrutinib are being evaluated and other BTK inhibitors (acalabrutinib, zanubrutinib) are also being tested, including a randomized study of obinutuzumab with or without zanubrutinib (NCT03332017)..$^{56,57}$ It is hoped that these studies will clarify the potential role of BTK inhibitors in patients with FL.

\section{Inhibitors of the mTOR pathway: a missed opportunity?}

It is also noteworthy that the mTOR pathway is dysregulated in a substantial proportion of cases of FL, particularly those with mutations activating RRAGC, $A T P 6 V 1 B 2$ and ATP6AP1 and inactivating the upstream regulator SESTRIN1. The oral MTORC1 inhibitor everolimus was found to be active with an overall response rate of $61 \%$ (and a median response duration of 11.5 months) (Table 1)..$^{58}$ Temsirolimus, which requires intravenous administration, achieved overall and complete response rates of $54 \%$ and $26 \%$, respectively, and the median PFS was 13 months. ${ }^{59}$ Of note, these clinical results were observed prior to our knowledge of the molecular alterations involving this pathway, and it would be interesting to evaluate whether their presence is associated with higher efficacy of $\mathrm{mTOR}$ inhibitors.

\section{The unexpected results of BCL2 inhibitors}

With persistent BCL2 expression linked to the hallmark $t(14: 18)$ translocation, one would expect that BCL2 inhibitors would demonstrate activity in FL. In the initial phase I study, response to single-agent venetoclax was observed in $38 \%$ of 29 patients (with $17 \%$ reaching a complete response), and the median PFS and duration of response were 11 and 27 months, respectively (Table 1). ${ }^{60}$ Based on these preliminary results, venetoclax was combined with rituximab (single-arm study) or with B-R (randomized phase II study) to gauge its clinical value. Results demonstrated a lack of significant benefit from the addition of venetoclax to B-R (while toxicity was significantly increased) and only modest benefit when venetoclax was added to rituximab. ${ }^{61}$ Several hypotheses for this limited activity have been raised, including: (i) the possibility that BCL2-deregulated expression is critical in early steps of the development of FL, but that tumor cells subsequently acquire other genetic alterations and no longer depend on BCL2 for their survival; (ii) the role of alternative actors of the apoptotic machinery (BCLX-L) in protecting these cells from dying; and (iii) mutations in the BCL2 gene that can impair venetoclax binding. ${ }^{62}$

\section{Epigenetic therapies}

Mutations in genes involved in chromatin organization appear as early founding events in germinal center B-cell lymphomagenesis. FL relies on epigenetic dysregulation to allow maintenance of a germinal center phenotype, thereby facilitating cell survival and proliferation. 
Epigenetic changes also contribute to immune dysregulation via suppression of major histocompatibility complex expression and tumor infiltrating lymphocyte content.

Tazemetostat represents the most successful epigenetic therapy to date in FL. ${ }^{63}$ Activating mutations in EZH2 occur in about $20 \%$ of $\mathrm{FL}$, and result in aberrant silencing of differentiation genes, allowing continued survival and proliferation of FL (Figure 2). Tazemetostat inhibits EZH2, allowing for normal transcription of repressed genes. In phase I and II studies evaluating tazemetostat monotherapy, the overall response rate was $69 \%$ in EZH2-mutant FL, with a median PFS of 13.8 months. Tazemetostat also demonstrated activity in EZH2 wildtype FL with an overall response rate of $35 \%$ and PFS of 11.1 months. Tazemetostat was well tolerated, with only $4 \%$ of patients suffering a serious treatment-related adverse event, a favorable profile for combination therapies. Other EZH2 inhibitors are in early development.

Other epigenetically targeted agents are also under investigation. Histone deacetylase inhibitors counteract the epigenetic impact of CREBBP and EP300 mutations (Figure 2), and have demonstrated activity in FL. Four phase II studies have documented response rates of 47 $64 \%$ with abexinostat or vorinostat, but with significant hematologic and gastrointestinal tract toxicity. ${ }^{64-67}$ In each of these studies, higher response rates to histone deacetylase inhibition were seen in FL than in other types of nonHodgkin lymphoma, suggesting a particular sensitivity to epigenetic modulation. As yet no studies have evaluated whether mutations in EP300 or CREBBP predict for response in $\mathrm{FL}$, and this may be worth exploring; however, it is interesting to note that in a study of the histone deacetylase inhibitor panobinostat in diffuse large B-cell lymphoma, CREBBP or EP300 mutations were not associated with response. ${ }^{68}$ Additional in vivo work suggests that inhibitors of the demethylase KDM5 may restore normal histone methylation and gene expression in KMT2Dmutant disease, another gene commonly affected in FL. ${ }^{69}$

\section{Immunotherapies: early successes and promising potential}

\section{Single-agent anti-CD20 therapy remains viable for selected patients}

In patients with relapsed disease and low tumor burden following initial anti CD20-containing therapy, re-challenge with monoclonal antibodies, including rituximab

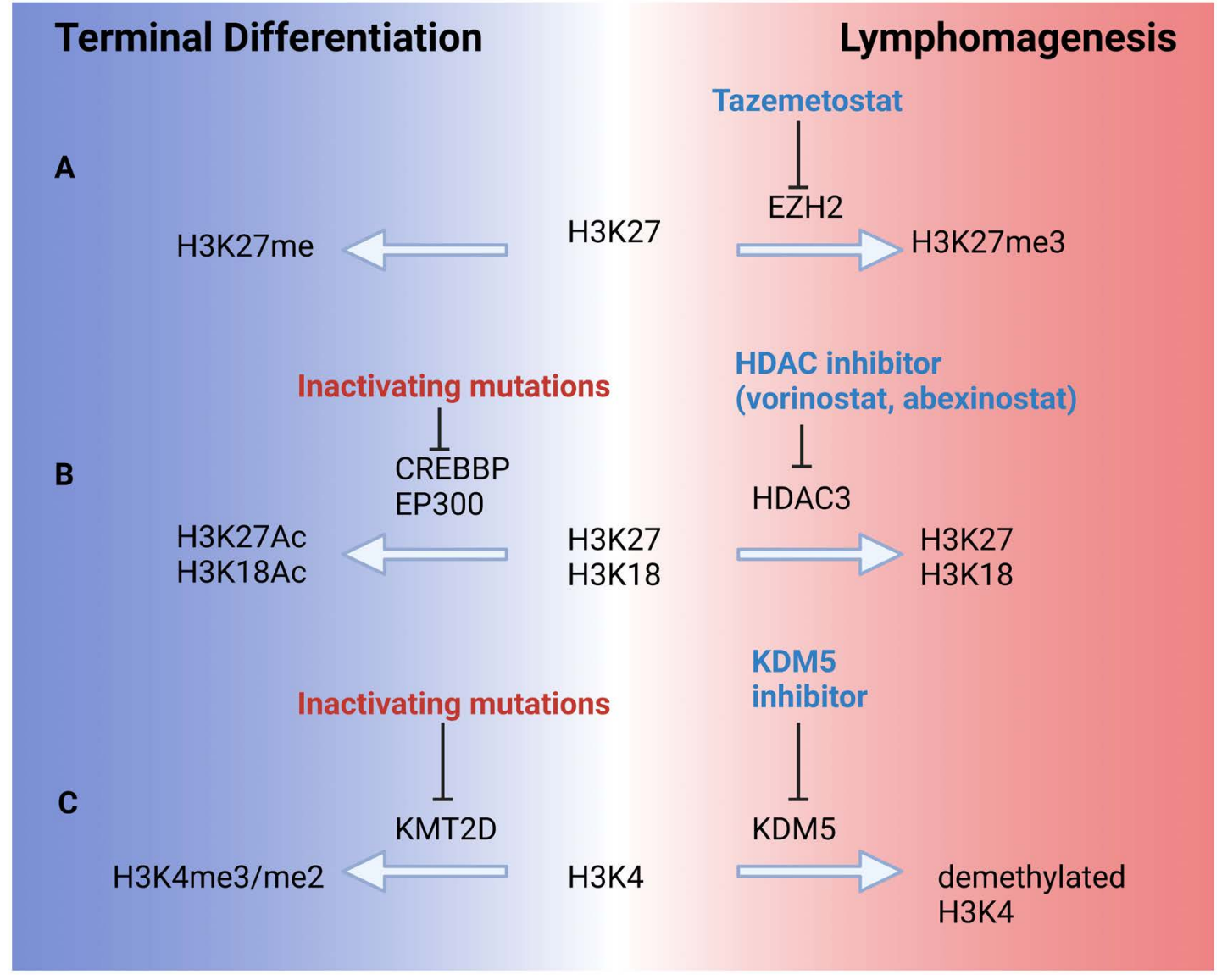

Figure 2. Epigenetic dynamics in follicular lymphoma. Frequent epigenetic mutations in follicular lymphoma result in repression of mature B-cell differentiation factors and cell regulators, preventing terminal differentiation and exit from the cell cycle. This allows a germinal center phenotype, genomic instability, and cellular proliferation to persist. (A) Activating mutations in EZH2 result in aberrant trimethylation of lysine 27 in histone 3 (H3K27me3), resulting in transcriptional silencing and lymphomagenesis. Tazemetostat inhibits EZH2, restoring normal H3K27 methylation. (B) Loss-of-function mutations in the histone acetyltransferases CREBBP and EP30O result in decreased acetylation of histones H3K27 and H3K18, shifting the balance toward histone deacetylation by HDAC3. Histone deacetylase inhibitors prevent this deacetylation. (C) KMT2D is a histone methyltransferase and is frequently inactivated in follicular lymphoma, favoring demethylation of H3K4 and repression of key differentiation genes. Inhibitors of the demethylase KDM5 restore normal H3K4 methylation and gene expression in KMT2D-mutant lymphoma. HDAC: histone deacetylase. 
and obinutuzumab, remains an option. Prior sensitivity to anti-CD20 treatment is essential when considering this option. Sensitivity has classically been defined as having a response to anti-CD20 treatment lasting at least 6 months. $^{70-72}$

Several studies have evaluated anti-CD20 monotherapy re-challenge in the second or later line of therapy. A phase II trial of rituximab re-challenge in patients with relapsed indolent non-Hodgkin lymphoma (92\% FL) demonstrated an overall response rate of $40 \%$, with $11 \%$ attaining complete responses and the median time to progression being 17.8 months. ${ }^{73}$ A case-control study demonstrated response rates of $76 \%$ with second-line rituximab monotherapy in FL, and a median PFS of 19.2 months. ${ }^{74}$ In the RESORT clinical trial, patients with low tumor burden FL with initial response to rituximab monotherapy were randomized to maintenance rituximab or re-treatment with rituximab at progression of disease, with patients in the re-treatment cohort demonstrating response rates of $61 \%$ at first re-treatment and $67 \%$ at second re-treatment. ${ }^{70}$

With regard to the choice of anti-CD20 agent, obinutuzumab has not been proven to be significantly superior to rituximab, but it can be used in patients with adverse reactions to rituximab, while ofatumumab appears inferior to rituximab. ${ }^{71,75}$

We consider single-agent rituximab (or rarely obinutuzumab) a useful option in instances of mild tumor burden where an indication to treat nonetheless exists and when rituximab-based combinations - providing deeper and more durable responses - are not suitable.

\section{Immunomodulators}

Lenalidomide is an immunomodulatory agent that functions via cereblon-mediated ubiquitination and degradation of target transcriptional factors, resulting in potential direct cellular toxicity to lymphoma cells as well as potentiation of immune effector cells in the tumor microenvironment. Lenalidomide has demonstrated synergy with anti-CD20 antibodies such as obinutuzumab and rituximab, via reconstitution of a functional immune synapse, improved natural killer cell function, and greater antibody-dependent cellular cytotoxicity. ${ }^{76-78}$ This benefit was demonstrated in the phase III AUGMENT trial, which evaluated rituximab with or without lenalidomide in relapsed rituximab-sensitive FL, and found a significant PFS benefit with the combination of lenalidomide plus rituximab (R2). ${ }^{72}$ A post-hoc analysis also indicated an OS benefit for the combination, although patients in both study arms had very favorable outcomes (2-year OS of $86 \%$ and $95 \%$ in the rituximab/placebo and $\mathrm{R} 2$ arms, respectively), likely reflecting the select charactersitics of the study population. Of note, infections, neutropenia and skin rashes were more frequently encountered in patients receiving $\mathrm{R} 2$. Based on these results, and given the similar efficacy of this combination when compared with classical immunochemotherapy in the first-line setting (as demonstrated in the RELEVANCE study), R2 is now a standard of care for relapsed/refractory, rituximabsensitive FL following anti-CD20 or chemoimmunotherapy. ${ }^{25}$ A single-arm, phase II study of obinutuzumab and lenalidomide in relapsed/refractory disease, including rituximab-refractory FL, showed similarly high response rates. $^{79}$

Immunomodulators have intriguing potential in terms of synergy, particularly with other immunotherapies, as already evidenced with rituximab and obinutuzumab. Further studies evaluating the addition of immunotherapies to the R2 or obinutuzumab-lenalidomide backbone are underway. For example, a phase Ib/II study evaluated the use of atezolizumab combined with obinutuzumablenalidomide in patients who had received at least one prior line of immunochemotherapy, with encouraging rates of durable clinical responses (3-year PFS 68.4\%, 3year OS 90\%). ${ }^{80}$ Another randomized study is evaluating the combination of R2 with tafasitamab, a CD19-directed Fc-engineered antibody, which had modest activity as a single agent in $\mathrm{FL}$, but produced encouraging response rates in diffuse large $\mathrm{B}$-cell lymphoma when combined with lenalidomide (NCT04680052) ${ }^{81}$

\section{Cellular therapies}

Recently, the Food and Drug Administration in the USA approved axicabtagene ciloleucel as the first CAR T-cell therapy for FL, based on results of the ZUMA-5 trial. ${ }^{82}$ This study evaluated axicabtagene ciloleucel CAR T-cell therapy in patients with indolent non-Hodgkin lymphoma, including 127 with FL, who had relapsed/refractory disease after two or more prior lines of therapy, including anti-CD20 and alkylator-containing therapy. The overall response rate was $94 \%$ and complete response rate $79 \%$ for FL patients; at 18 months PFS was $69 \%$ and OS was $88 \%$ (Table 2). Similar response rates, but a decreased PFS rate at 18 months, were noted in patients with POD24 disease compared to those without (55\% vs. $84 \%$, respectively). ${ }^{83}$ The results of this trial have been compared to those of an external propensity scorematched cohort of patients not enrolled in the study who otherwise met criteria for ZUMA-5, showing significant improvement in overall response rate, PFS, time to next treatment, and OS with CAR T-cell therapy compared to alternative therapies. ${ }^{84}$ Low-grade cytokine release syndrome occurred in the majority of patients with FL, while $6 \%$ experienced grade $\geq 3$ cytokine release syndrome; notably, $50 \%$ of patients received tocilizumab during therapy, indicating that close monitoring, similar to that necessary with large cell lymphoma, will still be required. For patients with FL, the frequency $(56 \%)$ and severity (15\% with grade $\geq 3$ events) of neurological events, and their duration (median of 14 days), represent a significant hurdle for broader application of this therapy.

Interestingly, re-treatment with axicabtagene ciloleucel in patients who relapsed after initial therapy with this drug also generated favorable responses. Thirteen patients (11 with FL) who had undergone initial axicabtagene ciloleucel therapy, as per ZUMA-5, and achieved an initial response followed by progression, were treated with repeated axicabtagene ciloleucel administration after it had been confirmed that CD19 expression persisted on FL cells. The response rate was $100 \%$ with $77 \%$ achieving a complete response, and $58 \%$ being progression-free at 12 months after re-treatment.

Evaluation of other CAR T-cell products is also underway. A phase II study of tisagenlecleucel in relapsed/refractory $\mathrm{FL}$ after two or more lines of prior therapy is ongoing. ${ }^{85}$ At the most recent evaluation, there was a high overall response rate of $86 \%$ (95\% CI: 56 $75 \%$ ) with a complete response rate of $66 \%$ (95\% CI: 78$92 \%$ ) and PFS at 6 months of $76 \%$ (95\% CI: $65-84 \%)$. A favorable toxicity profile was noted with cytokine release 
Table 2. Selected chimeric antigen receptor T-cell and bispecific antibody trials and outcomes.

\begin{tabular}{|c|c|c|c|c|c|c|c|c|c|}
\hline CAR T-cell product & Phase & $\begin{array}{l}\text { Prior lines } \\
\text { of treatment }\end{array}$ & $\begin{array}{l}\text { N. of patients } \\
\text { with FL }\end{array}$ & $\begin{array}{l}\text { ORR } \\
(\%)\end{array}$ & $\begin{array}{l}\text { CRR } \\
(\%)\end{array}$ & $\begin{array}{c}\text { PFS } \\
\text { (time point, \%) }\end{array}$ & $\begin{array}{c}\text { DoR } \\
\text { (time point, \%) }\end{array}$ & $\begin{array}{c}\text { CRS, \% } \\
\text { (grade III-IV) }\end{array}$ & $\begin{array}{l}\text { ICANS, \% } \\
\text { (grade III-V) }\end{array}$ \\
\hline Axicabtagene ciloleuce ${ }^{82}$ & II & $\geq 2$ & 84 & 94 & 79 & $18 \mathrm{mo}, 69 \%$ & $18 \mathrm{mo}, 69 \%$ & $78(6)$ & $56(15)$ \\
\hline Tisagenlecleucel $^{85}$ & II & $\geq 2$ & 94 & 86 & 66 & $6 \mathrm{mo}, 76 \%$ & N/A & $48.5(0)$ & $9.3(1.0)$ \\
\hline Bispecific anthody & Phase & $\begin{array}{l}\text { Prior lines } \\
\text { of treatment }\end{array}$ & $\begin{array}{c}\text { N. of patients } \\
\text { with FL }\end{array}$ & $\begin{array}{l}\text { ORR } \\
(\%)\end{array}$ & $\begin{array}{l}\text { CRR } \\
(\%)\end{array}$ & $\begin{array}{c}\text { PFS, } \\
\text { median (mo) }\end{array}$ & $\begin{array}{c}\text { DoR, } \\
\text { median (mo) }\end{array}$ & $\begin{array}{c}\text { CRS, \% } \\
\text { (grade III-IV) }\end{array}$ & $\begin{array}{l}\text { ICANS, \% } \\
\text { (grade III-V) }\end{array}$ \\
\hline Mosunetuzumab $b^{92}$ & I & $\geq 2$ & 62 & 68 & 50 & 11.8 & 20.4 & $23(1.6)$ & $45(0)$ \\
\hline Glofitamab $^{93}$ & I & $\geq 1$ & 44 & 71 & 48 & 11.8 & 10.8 & $50.3(3.5)^{*}$ & $3.5(1.2)^{*}$ \\
\hline Epcoritamab $^{94}$ & I & $\geq 1$ & 12 & 90 & 50 & N/A & $\mathrm{N} / \mathrm{A}$ & $59(0)^{*}$ & $8(4) *$ \\
\hline Odronextamab ${ }^{95}$ & I & $\geq 1$ & 28 & 93 & 75 & 12.8 & 7.7 & $62.2(7.1)^{*}$ & $(3.9)^{*}$ \\
\hline Anti-CD47 anthody & Phase & $\begin{array}{l}\text { Prior lines } \\
\text { of treatment }\end{array}$ & $\begin{array}{l}\text { N. of patients } \\
\text { with FL }\end{array}$ & $\begin{array}{l}\text { ORR } \\
(\%)\end{array}$ & $\begin{array}{l}\text { CRR } \\
(\%)\end{array}$ & $\begin{array}{c}\text { PFS, } \\
\text { median (mo) }\end{array}$ & $\begin{array}{c}\text { DoR, } \\
\text { median (mo) }\end{array}$ & $\begin{array}{l}\text { Anemia, \% } \\
\text { (grade III-IV) }\end{array}$ & \\
\hline Magrolimab $^{97}$ & $\mathrm{Ib} / \mathrm{II}$ & $\geq 1$ & 28 & $66^{*}$ & $24^{*}$ & N/A & $\mathrm{N} / \mathrm{A}$ & $27(15)$ & \\
\hline
\end{tabular}

*All patients with non-Hodgkin lymphoma, those with follicular lymphoma not reported individually. Data were collected from published papers and abstracts; comparisons cross trials are not possible because of the different eligibility criteria and other differences. FL: follicular lymphoma; ORR: overall response rate; CRR: complete response rate; PFS: progression-free survival; DoR: duration of response; CRS: cytokine release syndrome; ICANS: immune effector cell associated neurotoxicity; N/A: not available or not reported; mo: months.

syndrome occurring in $49 \%$ (all grade 2 or below) and neurotoxicity in $9 \%$ (all grade 2 or below, apart from a single grade 4 event which was self-limited). Another study is evaluating the activity of lisocabtagene maraleucel in the same setting (NCT04245839).

An array of clinical trials is in development with the aim of improving CAR T-cell therapy in B-cell lymphoma. ${ }^{86}$ Possible avenues include the use of combination products such as BTK inhibitors and checkpoint inhibitors, bispecific CAR targeting multiple lymphoma epitopes, and armored CAR products intended to overcome the immunosuppressive tumor microenvironment.

The current CD19-directed CAR T-cell results are encouraging, although longer-term data will be necessary to define the ultimate value of these treatments. There would be obvious appeal if this single-administration therapy produced prolonged disease-free remissions in a significant proportion of patients. Additionally, mitigation of cytokine release syndrome and neurotoxicity, either via supportive measures or less toxic CAR T-cell products, would broaden the applicability of these therapies, particularly if they can be administered in the outpatient setting. Assuming long-term efficacy is established, and with improvements in the avoidance and management of toxicities, CAR T cells will represent an appealing option in the therapeutic algorithm.

\section{Antibody-drug conjugates}

Antibody-drug conjugates enable targeted delivery of potent cytotoxic drugs to tumor cells, minimizing systemic toxicity. Such agents, targeting common B-cell antigens, have been developed across the spectrum of B-cell lymphomas, with polatuzumab vedotin and loncastuximab tesirine recently approved for patients with diffuse large B-cell lymphoma. ${ }^{87,88}$ Polatuzumab vedotin targets the $\mathrm{CD} 79 \mathrm{~b}$ antigen and has shown promising response rates alone or in combination with rituximab for patients with FL (overall response rate $70 \%$, complete response rate $45 \%){ }^{89}$ However, high rates of cumulative peripheral neuropathy were noted when prolonged cycles were administered, a significant concern in the context of the chronicity of FL. Furthermore, when combined with B-R in a randomized phase II study, polatuzumab did not improve the complete response rate or PFS for patients with FL, in contrast to the observations made in patients with diffuse large B-cell lymphoma. ${ }^{87}$ Several combinations of polatuzumab with other agents active in FL are currently being investigated..$^{0}$ The CD19-directed antibody loncastuximab tesirine also showed activity in 14 patients with FL with an overall response rate of $79 \%$ and a complete response rate of $64 \% .^{91}$ Combination studies with rituximab (NCT04998669) and a comparison of this agent versus idelalisib (NCT04699461) are planned.

\section{Bispecific antibodies}

A novel approach to lymphoma immunotherapy has been the use of bispecific T-cell engager therapies, which incorporate a CD3-binding component that engages $\mathrm{T}$ cells and a second tumor antigen-binding domain, most commonly CD20 in B-cell lymphoma. These agents function to induce activation and cytotoxic activity of $\mathrm{T}$ cells against CD20-expressing lymphoma cells. Like autologous CAR T-cell therapy, bispecific $\mathrm{T}$-cell engagers recruit the patient's native $T$ cells to target lymphoma, with the advantage of being an "off-the-shelf" option that bypasses the time and logistical challenges entailed in genetic modification of $\mathrm{T}$ cells. Early-phase clinical trials have demonstrated very encouraging results, with high response rates of $68-90 \%$ in patients with relapsed/refractory $\mathrm{FL}$, including complete responses in the majority of patients (Table 2). ${ }^{92-95}$ Limited follow-up is currently available to evaluate response duration, although preliminary data demonstrated durable responses lasting at least 18 months in patients having achieved a complete response after completion of therapy. Toxicities of concern include cytokine release syndrome and neurotoxicity, similar to the toxicities of CAR T-cell therapy, although these events were only observed during the initial one or two infusions, and both rates and severity were lower than those with CAR T-cell therapies. Further mitigation of toxicity may be possible via step-up dosing and/or subcutaneous administration. If durable responses are demonstrated and toxicity proves manageable, this class of agents appears to hold very significant promise in FL. Combination studies with other immune interventions or cytotoxic agents are under development (NCT04712097, NCT04663347, NCT04246086) and are evaluating the use of bispecific therapy in earlier phases of FL treatment. 


\section{Phagocytosis checkpoint inhibitors}

Macrophage-mediated phagocytosis is modulated by a series of checkpoints including the CD47-SIRP $\alpha$ checkpoint, classically known as the "don't eat me signal." Immunotherapies inhibiting this interaction facilitate macrophage activation and phagocytosis, and show promise in FL. Magrolimab, a humanized IgG4 isotype that binds CD47 and blocks interaction with SIRP $\alpha$, was evaluated in combination with rituximab in a phase Ib/II study of relapsed/refractory indolent non-Hodgkin lymphoma ( 28 of 29 patients with FL), with an overall response rate of $66 \%$ and a median duration of response not reached (range, 6.2-22.6 months). ${ }^{97}$ Toxicities included infusion reactions and a class toxicity with first-dose anemia, related to splenic phagocytosis of senescent red blood cells. Other CD47-targeted agents such as ALX148 and TTI-622 have also demonstrated activity in FL. ${ }^{98,99}$ In the preclinical setting, bispecific antibodies targeting CD47 and CD19 have been designed to increase drug specificity and decrease on-target, off-tumor toxicity ${ }^{100}$ Outside of the CD47-SIRPa axis, inhibitors of adenosine receptors have been shown to disinhibit antibody-dependent cellular phagocytosis, and show promise in preclinical studies.$^{101}$

As with other agents, there is increased interest in combining magrolimab or alternative pro-phagocytic agents with other immune therapies. A study showed that T-cell deficiency in mice abrogated the response to anti-phagocytic agents, and suggested that $\mathrm{CD} 8^{+}$cytotoxic $\mathrm{T}$ cells play an important role in the mechanism of action of prophagocytic therapies. ${ }^{102} \mathrm{CD} 47$ blockade also enhances the ability of dendritic cells to cross-prime $\mathrm{T}$ cells. ${ }^{102}$ Given these findings, there is a mechanistic rationale for the addition of a checkpoint inhibitor or T-cell co-stimulatory agent to anti-CD47 therapy. There is also in vitro evidence that chemotherapy administered prior to phagocytosis checkpoint inhibitors improves tumor response and augments host memory response against relapsing tumors. Further studies of combination therapies are needed, as well as a better basic understanding of the interactions between the innate and adaptive immune responses to lymphoma. ${ }^{102}$

\section{Checkpoint inhibitors and T-cell co-stimulatory agents}

The immune infiltrate in FL is enriched in $\mathrm{PD}$-1-positive immune cells, suggesting that immune tolerance plays a key role in lymphomagenesis, and that disruption of these signals via checkpoint blockade would be efficacious. ${ }^{103}$ Prior studies have investigated mechanisms of Tcell rescue via checkpoint blockers targeting the PD-1/PDL1 axis. ${ }^{104-108}$ While preliminary data suggested encouraging response rates from this treatment in combination with anti-CD20 monoclonal antibodies, no definitive benefit has been established, and single-agent activity has been disappointing, with response rates of $4-9 \%$.

There has also been interest in the utility of co-stimulatory agents in T-cell reconstitution and immunotherapy.

Table 3. Selected pivotal trials in relapsed or refractory follicular lymphoma.

\begin{tabular}{|c|c|c|c|c|c|c|c|}
\hline Targeted agents & Mechanism & Phase & Druggs & $\begin{array}{l}\text { Prior lines } \\
\text { of therapy }\end{array}$ & $\begin{array}{c}\text { N. of } \\
\text { planned } \\
\text { participants }\end{array}$ & Status & NCT \# \\
\hline Parsaclisib & PI3K $\delta$ inhibitor & III & $\begin{array}{l}\text { Parsaclisib + anti-CD20 vs. } \\
\text { Anti-CD20 monotherapy }\end{array}$ & $\geq 1$ & 416 & $\begin{array}{l}\text { Not yet } \\
\text { recruiting }\end{array}$ & NCT04796922 \\
\hline Zandelisib & PI3K $\delta$ inhibitor & II & Zandelisib monotherapy & $\geq 2$ & 180 & Recruiting & NCT03768505 \\
\hline Ibrutinib & BTK inhibitor & III & $\begin{array}{l}\text { Ibrutinib + R-CHOP or BR } v s . \\
\text { R-CHOP or BR }\end{array}$ & $\geq 1$ & 403 & Active, not recruiting & NCT01974440 \\
\hline \multirow[t]{2}{*}{ Zanubrutinib } & \multirow[t]{2}{*}{ BTK inhibitor } & & $\begin{array}{l}\text { Zandelisib - rituximab vs. } \\
\text { R-CHOP or BR }\end{array}$ & & 534 & Recruiting & \\
\hline & & $\begin{array}{c}\text { II } \\
\text { (randomized) }\end{array}$ & $\begin{array}{l}\text { Zanubrutinib + obinutuzumab vs. } \\
\text { Obinutuzumab }\end{array}$ & $\geq 2$ & 210 & Recruiting & NCT03332017 \\
\hline Tazemetostat & EZH2 inhibitor & III & Tazemetostat + R2 vs. R2 & $\geq 1$ & 518 & Recruiting & NCT04224493 \\
\hline Abexinostat & pan-HDAC inhibitor & II & Abexinostat monotherapy & $\geq 3$ & 139 & Active, not recruiting & NCT03600441 \\
\hline
\end{tabular}

\begin{tabular}{|c|c|c|c|c|c|c|c|}
\hline Immunotherapies & & & & & & & \\
\hline Magrolimab & Anti-CD47 MoAb & II & Magrolimab + rituximab & $\geq 1$ & 422 & Recruiting & NCT02953509 \\
\hline Mosunetuzumab & $\begin{array}{c}\text { Anti-CD20 x } \\
\text { anti-CD3 bispecific } \\
\text { antibody }\end{array}$ & III & $\begin{array}{l}\text { Mosunetuzumab + lenalidomide } v \text { s. } \\
\text { Rituximab + lenalidomide }\end{array}$ & $\geq 1$ & 400 & Not yet recruiting & NCT04712097 \\
\hline Tafasitamab & Anti-CD19 MoAb & III & Tafasitamab + R2 vs. R2 & $\geq 1$ & 618 & Recruiting & NCT04680052 \\
\hline Tisagenlecleucel & Anti-CD19 CAR T & II & Phase II study (ELARA) & $\geq 2$ & 97 & Active, not recruiting & NCT03568461 \\
\hline
\end{tabular}

\section{Other}

(177Lu)-Lilotomab

satetraxetan

Radiolabeled

(177 Lutetium) Anti-CD37 MoAb

Loncastuximab Anti-CD19 antibody

tesirine drug conjugate
IIb

(177Lu)-Lilotomab satetraxetan

$\geq 2$

II

Loncastuximab tesirine $v$. Idelalisib

$\geq 2$
This list is not exhaustive but outlines the agents discussed in the review. NCT: National Clinical Trials: PI3K: phosphoinositide 3-kinase; BTK: Bruton tyrosine kinase; R-CHOP: rit uximab, cyclophosphamide, doxorubicin, vincristine, prednisone; BR: bendamustine and rituximab; EZH2: enhancer of zeste homolog 2; R2: rituximab and lenalidomide; R: rituximab; HDAC: histone deacetylase; MoAb: monoclonal antibody; CAR T: chimeric antigen receptor T cell. 
The co-stimulatory receptor $4-1 \mathrm{BB}$ (CD137) is found on many activated immune cell subtypes. Activation of $4-1 \mathrm{BB}$ is associated with increased T-cell proliferation, survival, cytokine production, and functional maturation. ${ }^{109}$ Resident T cells in FL have been shown to express 4-1BB at high levels, providing a biological rationale for 4-1BB agonist therapy. ${ }^{109}$ However, early clinical trials showed only modest anti-tumor activity, with the therapeutic index limited by dose-related hepatotoxicity. ${ }^{110-112}$ It is thought that the hepatotoxicity seen in these studies was driven by offtumor Fc-mediated 4-1BB cross-linking by Fc $\gamma$ RIIb expressing liver resident cells. ${ }^{1{ }^{13}}$ While there remains a biological basis for 4-1BB co-stimulation in FL, a more tumor-specific mechanism is probably required. A possible solution is the use of bispecific therapies, which fuse 4-1BBL to a lymphoma-specific target such as CD19, minimizing off-target toxicity. This approach has shown synergistic activity with $\mathrm{CD} 3 / \mathrm{CD} 20$-targeted bispecific therapy in vitro and in vivo, and clinical trials are underway (NCT04077723). ${ }^{114}$

\section{Sequencing therapies in patients with relapsed/refractory follicular lymphoma}

When and how to utilize the growing array of therapies at our disposal remains a significant challenge. It is important to note that observation in many cases of relapsed or refractory FL remains a viable option if symptom burden is low and no absolute indications for treatment are met, such as the Groupe d'Etude des Lymphomes Folliculaires (GELF) criteria. As shown above, the number of options available for the management of patients requiring second and subsequent lines of therapy has increased markedly in the last decade, and newcomers are emerging. Several pivotal trials are under development, assessing the therapeutic value of single agents or various combinations of active compounds, and emphasizing the probable expansion of the field (Table 3). However, the lack of head-to-head evaluations of recently approved agents, and the usual pitfalls of cross-trial comparisons, represent obstacles to evidence-based decisions.

\section{Second-line treatment}

While there is no formal standard of care, the management algorithm (Figure 3) is relatively straightforward, since beside rituximab, alone or in combinations with lenalidomide, immunochemotherapy remains the principal option recommended by the National Comprehensive Cancer Network and European Society for Medical Oncology guidelines in the absence of histological transformation. These options achieve a clinical response in the majority of patients, which can be sustained for years. Choices among the different cytotoxic regimens and anti-CD20 antibodies have already been discussed above, as has the role of consolidation with anti-CD20 maintenance or ASCT.

In addition to the AUGMENT trial, the results of the front-line RELEVANCE study, which demonstrated comparable efficacy between R2 and immunochemotherapy, reinforce the efficacy of $\mathrm{R} 2$, and this regimen should be strongly considered if immunochemotherapy was used in the first line of treatment. ${ }^{25}$ Advantages of this approach include using a strategy with a different mode of action and leaving an open space for initiating immunochemotherapy (with eventual consolidation) later in the management of FL.

Beyond prior therapy and disease characteristics, the patient's ability to tolerate therapy must be considered. In patients of advanced age, who are frail, or have comorbidities, options include rituximab as a single agent or in combination with lenalidomide (with the appropriate dose adaptations according to kidney function), alkylating agents or localized radiation therapy. A personalized approach is recommended, and it remains possible to

\section{2nd line ( $1^{\text {st }}$ progression)}

CONSIDER

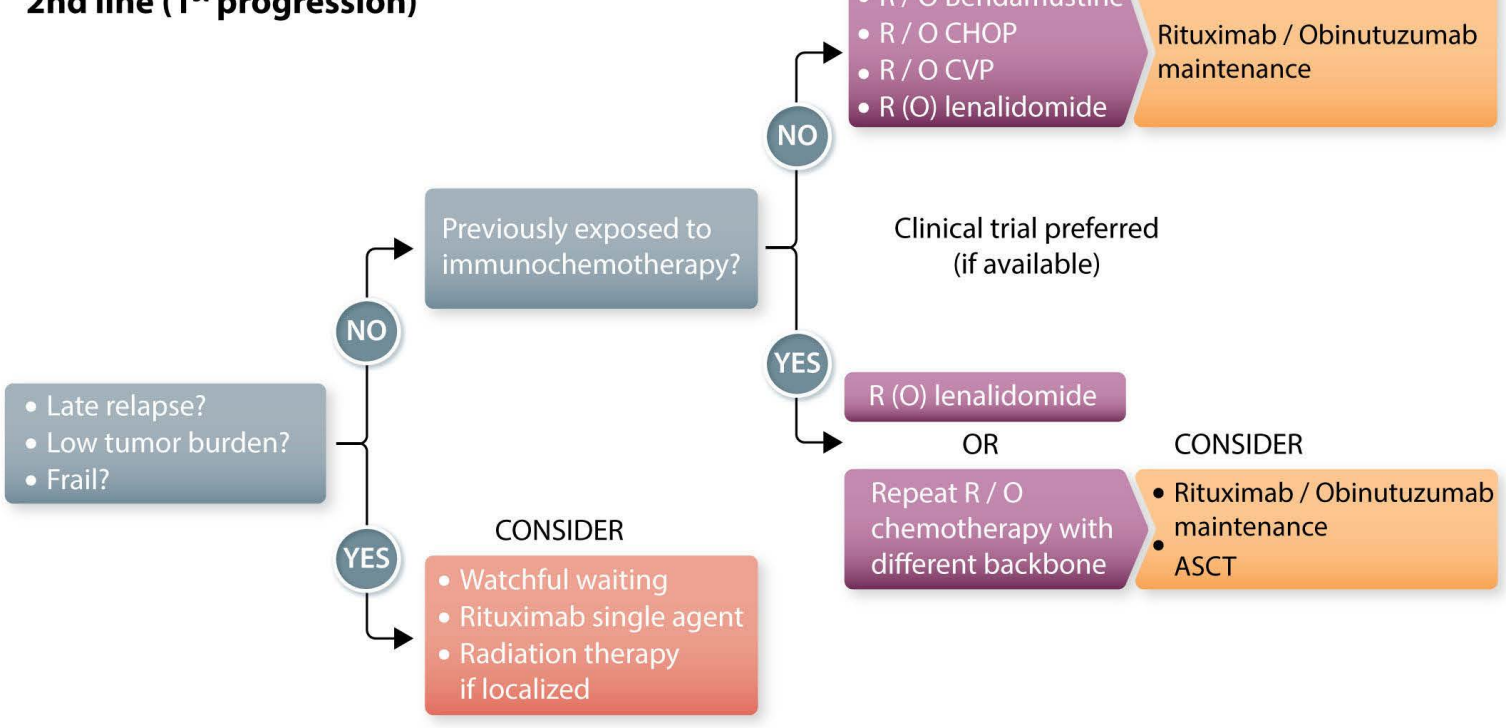

Always rule out histological transformation - new biopsy recommended

Figure 3. Schema for treatment choice at the time of first disease progression. After ruling out histological transformation, the choice of agent depends on prior therapy, the timing of relapse, tumor burden, and patients' ability to tolerate therapy. R: rituximab; O: obinutuzumab; CHOP: cyclophosphamide, adriamycin, vincristine, prednisone; CVP: cyclophosphamide, vincristine, prednisone: ASCT: autologous stem cell transplantation. 


\section{$3^{\text {rd }}$ line and + (subsequent progression)}

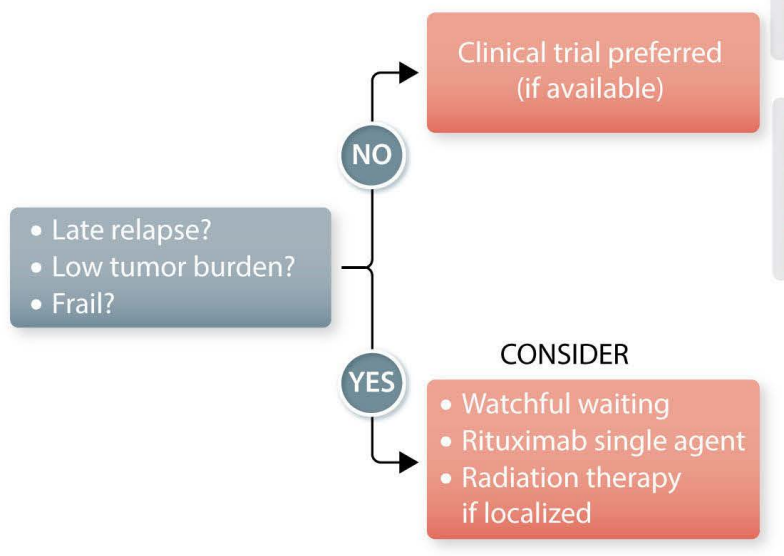

\section{Preferred options Alternative options}

More intensive (deeper/more prolonged response)

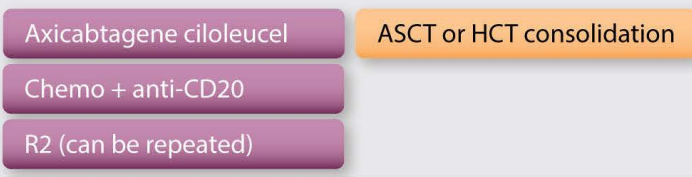

Less intensive therapy (chronic management)

PI3K inhibitor

Tazemetostat if EZH2 mut

Tazemetostat if EZH2 WT

Radioimmunotherapy

Rituximab (if free interval)

Palliative radiation

Always rule out histological transformation - new biopsy recommended

Figure 4. Schema for treatment choice in third-line and subsequent settings. Given the paucity of randomized data and large number of effective therapies in this setting, treatment is highly personalized, dependent on disease characteristics, prior therapies, and patients' ability to tolerate treatment. Clinical trials should always be strongly considered. ASCT: autologous stem cell transplantation; HCT: hematopoietic cell transplantation; R2: rituximab and lenalidomide; PI3K: phosphoinositide 3-kinase; mut: mutated; WT: wild-type.

offer substantial benefits for these patients while avoiding significant toxicity.

\section{Management in early progression}

In cases of early progression (i.e., EFS12 or POD24 disease), histological transformation should always be considered. A biopsy should be systematically performed in all cases to rule out such transformation; several clinical featues, such as lack of response during chemotherapy or rapid tumor growth, elevated serum lactate dehydrogenase levels, hypercalcemia, appearance of B symptoms, or rapid decline of the performance status are highly suggestive of histological transformation and might be used as surrogates when a biopsy is not feasible. ${ }^{3}$ If histological transformation is confirmed, in patients not previously exposed to anthracycline, R-CHOP often constitutes the standard of care. Alternative salvage chemotherapy regimens followed by ASCT are recommended for those previously exposed to anthracycline, or in those unresponsive to R-CHOP. Cellular therapy is an important emerging option for patients with histological transformation but unmet needs remain. ${ }^{5}$

If histological transformation is ruled out, several options should be considered. Standard options such as obinutuzumab-bendamustine and anti-CD20-lenalidomide combinations are often utilized, and their efficacy in POD24 patients has been demonstrated. ${ }^{20,72,79,115}$ If an adequate response is achieved, some centers advocate for ASCT consolidation in eligible patients, and this remains a viable option, particularly in younger, fit patients in whom durable response is desirable. Aside from chemoimmunotherapy and R2, other strategies appear to be active in patients with POD24, including some PI3K inhibitors, bispecific antibodies (such as mosunetuzum$\mathrm{ab}$ ), and CAR T cells. ${ }^{50,83,9,2,16}$ Decisions in this situation can remain challenging, and studies such as SWOG S1608
(NCT03269669) comparing obinutuzumab-chemotherapy versus obinutuzumab-lenalidomide or obinutuzumabumbralisib will be helpful to further elucidate the optimal management of these patients.

\section{Third-line setting and beyond}

In multiply relapsed and refractory disease, a broad array of effective options (Figure 4) remains available, although the choice of therapy is more individualized and less defined by randomized trials. Decisions depend primarily on patients' prior treatment, response to therapies, disease-related symptoms and tumor burden. The choice of drug is then based on relative efficacy (limited by cross-trial comparisons), toxicity profiles, response rates, and patients' priorities.

Repeating rituximab-based regimens with chemotherapy or lenalidomide is often feasible if a significant progression-free interval of at least 2 years was achieved with the last treatment. In cases of refractory disease or early progression, changing the class of agents is generally preferred.

The choice of agent is also dictated by the anticipated depth and duration of response. Oral agents such as PI3K or EZH2 inhibitors are often considered earlier in treatment despite the low complete response rates observed and the median PFS and duration of response close to 12-14 months. These agents may be better positioned when more efficacious strategies have been exhausted, or as temporary solutions before implementing a more effective therapy.

Beside the presence of an EZH2 mutation, there are no biological criteria available to guide us toward a given treatment option. If the patient presents with significant tumor bulk or significant disease-related symptoms, it is probably preferable to use regimens allowing a more rapid and sustained response. In fit patients, if adverse features (high FLIPI, bulky disease, B symptoms, short 
treatment-free interval) are present, and after having ruled out the possibility of histological transformation, strategies offering a higher response rate and prolonged PFS should be considered with the patient. While ASCT or allogeneic transplant were reasonable options in this setting (if not used before), the availability of CAR T cells despite the limited follow-up presently available - represents an important opportunity to consider prior to transplantation. For all patients, the consideration of a clinical trial is encouraged, given the rapid evolution in the field with effective agents and combinations. Overall, the risk/benefit ratio of each option should be assessed in the context of the patients' overall condition and their individual goals for therapy.

\section{Remaining gaps and perspectives}

Several questions remain unanswered regarding the optimal sequencing of new agents. Overall, we know little about how prior treatment affects disease biology, and hence influences the response to the next class of agent. It is suspected that the use of T-cell-depleting agents, such as bendamustine or purine analogues, may affect the quality of host $T$ cells and result in suboptimal results of CAR T-cell therapy, and possibly other immunotherapies such as immunomodulators and bispecific antibodies. Further translational and clinical evaluation regarding the impact of prior treatment on the mechanism of action and efficacy of subsequent treatments is needed.

Markers able to predict response to therapy are also lacking. The potential influence of specific molecular alterations has not been explored in the relapsed/refractory setting (except for higher response rates to tazemetostat when an EZH2 mutation is present). It is conceivable that alterations in the B-cell receptor signaling pathway or in the metabolic regulatory pathway may affect the efficacy of agents targeting those pathways. Likewise, mutations affecting MHC expression or T-cell subpopulation might potentially influence some immunotherapies. Further investigations in this area, such as circulating tumor DNA assays to characterize the mutational landscape, will be of interest.

Another important challenge is the design and clinical execution of more rational therapeutic combinations, and many possible examples have been outlined above. Phase II studies with early and standardized evaluation end- points, such as positron emission tomography/computed tomography response or circulating tumor DNA evaluation, are desirable. Despite the difficulty of aligning different stakeholders with potential competing interests, conducting drug development in parallel may allow a more direct comparison of the respective benefits of these different strategies. Evaluating patients' quality of life and assessing their potential preferences when facing options with different routes of administration, treatment durations and toxicity patterns fully deserves additional methodological efforts and investment.

Two major therapeutic mechanisms carry hope for future progress. Given the central role of epigenetic alterations in the development of FL, we anticipate that epigenetic modifiers will be able to eradicate - or substantially deplete - the pool of lymphoma clonal precursor cells, providing an avenue toward cure. Tazemetostat represents an important proof of concept, but the development of more efficacious agents remains in its infancy, and specificity and tolerability of epigenetic-directed drugs remain challenging. Immune-based therapies represent another area of significant potential, with promising results observed with cellular therapies and bispecific antibodies. It is likely that various immunotherapies will represent the backbone of future combinations and progress, as shown by multiple studies currently in development. While it remains uncertain whether harnessing immune cells to efficiently eliminate FL cells will let us envision the cure of this disease, the remarkable survival improvements achieved in the last 20 years with antiCD20 monoclonal antibodies allow us to continue to be optimistic.

\section{Disclosures}

In the last 12 months GS has received financial compensations for participating in advisory boards or consulting from: Abbvie, Bayer, Beigene, BMS/Celgene, Epizyme, Genentech/Roche, Genmab, Incyte, Janssen, Kite/Gilead, Loxo, Milteniy, Morphosys, Novartis, Rapt, Regeneron and Takeda.

\section{Contributions}

Both authors collected and analyzed data, wrote and approved the manuscript

\section{Funding}

This research was funded in part through the NIH/NCI Cancer Center support grant P30 CA008748.

\section{References}

1. Junlén H, Peterson S, Kimby E, et al. Follicular lymphoma in Sweden: nationwide improved survival in the rituximab era, particularly in elderly women: a Swedish Lymphoma Registry study. Leukemia. 2015;29(3):668-676.

2. Sarkozy C, Maurer MJ, Link BK, et al. Cause of death in follicular lymphoma in the first decade of the rituximab era: a pooled analysis of French and US cohorts. J Clin Oncol. 2019;37(2):144-152.

3. Al-Tourah AJ, Gill KK, Chhanabhai M, et al. Population-based analysis of incidence and outcome of transformed non-Hodgkin's lymphoma. J Clin Oncol. 2008;26(32):51655169.
4. Federico M, Barrigón MDC, Marcheselli L, et al. Rituximab and the risk of transformation of follicular lymphoma: a retrospective pooled analysis. Lancet Haematol. 2018;5(8):e359-367.

5. Smith S. Transformed lymphoma: what should I do now? Hematol Am Soc Hemato Educ Progr. 2020;2020(1):306-311.

6. Salles G. How do I sequence therapy for follicular lymphoma? Hematology Am Soc Hematol Educ Program. 2020;20(1):287-294.

7. Mozas P, Nadeu F, Rivas-Delgado A, et al. Patterns of change in treatment, response, and outcome in patients with follicular lymphoma over the last four decades: a singlecenter experience. Blood Cancer J. 2020;10 (3):31.

8. Pettengell R, Donatti C, Hoskin P, et al. The impact of follicular lymphoma on healthrelated quality of life. Ann Oncol. 2008;19 (3):570-576.

9. Kridel R, Chan FC, Mottok A, et al. Histological transformation and progression in follicular lymphoma: a clonal evolution study. PLoS Med. 2016;13(12):e1002197.

10. Pasqualucci L, Khiabanian H, Fangazio M, et al. Genetics of follicular lymphoma transformation. Cell Rep. 2014;6(1):130.

11. Montoto S, López-Guillermo A, Altés A, et al. Predictive value of Follicular Lymphoma International Prognostic Index (FLIPI) in patients with follicular lymphoma at first progression. Ann Oncol. 2004;15(10):14841489

12. Casulo C, Byrtek M, Dawson KL, et al. Early relapse of follicular lymphoma after ritux- 
imab plus cyclophosphamide, doxorubicin, vincristine, and prednisone defines patients at high risk for death: an analysis from the National LymphoCare Study. J Clin Oncol. 2015;33(23):2516-2522

13. Maurer MJ, Bachy E, Ghesquières $\mathrm{H}$, et al. Early event status informs subsequent outcome in newly diagnosed follicular lymphoma. Am J Hematol. 2016;91(11):10961101.

14. Moccia AA, Schär S, Hayoz S, et al. Prognostic value of POD24 validation in follicular lymphoma patients initially treated with chemotherapy-free regimens in a pooled analysis of three randomized trials of the Swiss Group for Clinical Cancer Research (SAKK). Br J Haematol. 2021;192 (6):1031-1034.

15. Binkley MS, Brady JL, Haij C, et al. Salvage treatment and survival for relapsed follicular lymphoma following primary radiotherapy: a collaborative study on behalf of ILROG. Int J Radiat Oncol Biol Phys. 2019;104 (3):522.

16. Casulo C, Barr PM. How I treat early-relapsing follicular lymphoma. Blood. 2019;133 (14):1540-1547.

17. Wagner-Johnston ND, Link BK, Byrtek M, et al. Outcomes of transformed follicular lymphoma in the modern era: a report from the National LymphoCare Study (NLCS). Blood. 2015;126(7):851-857.

18. Rummel M, Kaiser U, Balser C, et al. Bendamustine plus rituximab versus fludarabine plus rituximab for patients with relapsed indolent and mantle-cell lymphomas: a multicentre, randomised, openlabel, non-inferiority phase 3 trial. Lancet Oncol. 2016;17(1):57-66.

19. Sehn LH, Chua N, Mayer J, et al. Obinutuzumab plus bendamustine versus bendamustine monotherapy in patients with rituximab-refractory indolent nonHodgkin lymphoma (GADOLIN): a randomised, controlled, open-label, multicentre, phase 3 trial. Lancet Oncol. 2016;17(8):1081-1093.

20. Cheson BD, Chua N, Mayer J, et al. Overall survival benefit in patients with rituximabrefractory indolent non-hodgkin lymphoma who received obinutuzumab plus bendamustine induction and obinutuzumab maintenance in the GADOLIN study. J Clin Oncol. 2018;36(22):2259-2266.

21. Hiddemann W, Barbui AM, Canales MA, et al. Immunochemotherapy with obinutuzumab or rituximab for previously untreated follicular lymphoma in the GALLIUM study: Influence of chemotherapy on efficacy and safety. J Clin Oncol. 201836(23):2395-2404.

22. Link BK, Day B-M, Zhou X, et al. Secondline and subsequent therapy and outcomes for follicular lymphoma in the United States: data from the observational National LymphoCare Study. Br J Haematol. 2019:184(4):660-663.

23. Batlevi CL, Sha F, Alperovich A, et al. Follicular lymphoma in the modern era: survival, treatment outcomes, and identification of high-risk subgroups. Blood Cancer J. 2020;10(7):74.

24. Weide R, Feiten S, Friesenhahn V, et al. Retreatment with bendamustine-containing regimens in patients with relapsed/refractory chronic lymphocytic leukemia and indolent B-cell lymphomas achieves high response rates and some long lasting remissions. Leuk Lymphoma. 2013;54(8):16401646.

25. Morschhauser F, Fowler NH, Feugier P, et al.
Rituximab plus lenalidomide in advanced untreated follicular lymphoma. N Engl J Med. 2018;379(10):934-947.

26. Strati P, Samaniego F, Gallardo M, Nooran $M$. Treatment and outcome of patients with follicular lymphoma relapsed or progressed after frontline lenalidomide and rituximab. Blood. 2020;136(Suppl 1):31-32

27. Harrop S, Polliack A, Tam CS. Chronic lymphoproliferative disorders and secondary cancers in the era of purine analogues and beyond. Leuk Lymphoma. 2020;62(4):771 778.

28. Ghione P, Cavallo F, Visco C, et al. A retrospective study of R-DHAP/Ox for early progressing follicular lymphoma. Br J Haematol. 2018;183(5):828-831.

29. Martinelli G, Montoro J, Vanazzi A, et al. Chlorambucil-rituximab as first-line therapy in patients affected by follicular nonHodgkin's lymphoma: a retrospective single-centre study. Hematol Oncol. 2015;33 (4):129-135.

30. Peterson BA, Petroni GR, Frizzera G, et al. Prolonged single-agent versus combination chemotherapy in indolent follicular lymphomas: a study of the Cancer and Leukemia Group B. J Clin Oncol. 2016;21 (1):5-15.

31. Forstpointner R, Unterhalt M, Dreyling M et al. Maintenance therapy with rituximab leads to a significant prolongation of response duration after salvage therapy with a combination of rituximab, fludarabine, cyclophosphamide, and mitoxantrone ( $\mathrm{R}$ FCM) in patients with recurring and refractory follicular and mantle cell lymphomas: results of a prospective randomized study of the German Low Grade Lymphoma Study Group (GLSG). Blood. 2006;108(13):40034008

32. Van Oers MHJ, Van Glabbeke M, Giurgea L, et al. Rituximab maintenance treatment of relapsed/resistant follicular non-Hodgkin's lymphoma: long-term outcome of the EORTC 20981 phase III randomized intergroup study. J Clin Oncol. 2010;28(17):28532858

33. Vidal L, Gafter-Gvili A, Salles G, et al Rituximab maintenance improves overall survival of patients with follicular lymphoma - individual patient data meta-analysis. Eur J Cancer. 2017;76:216-225.

34. Schouten HC, Qian W, Kvaloy S, et al. Highdose therapy improves progression-free survival and survival in relapsed follicular nonHodgkin's lymphoma: results from the randomized European CUP trial. J Clin Oncol. 2003;21(21):3918-3927.

35. le Gouill S, de Guibert S, Planche L, et al. Impact of the use of autologous stem cell transplantation at first relapse both in naïve and previously rituximab exposed follicular lymphoma patients treated in the GELA/GOELAMS FL2000 study. Haematologica. 2011;96(8):1128-1135.

36. Jurinovic V, Metzner B, Pfreundschuh M, et al. Autologous stem cell transplantation for patients with early progression of follicular ymphoma: a follow-up study of 2 randomized trials from the German Low Grade Lymphoma Study Group. Biol Blood Marrow Transplant. 2018;24(6):1172-1179.

37. Casulo C, Friedberg JW, Ahn KW, et al Autologous transplantation in follicular lymphoma with early therapy failure: a National LymphoCare Study and Center for International Blood and Marrow Transplant Research analysis. Biol Blood Marrow Transplant. 2018;24(6):1163-1171

38. Pettengell R, Uddin R, Boumendil A, et al
Durable benefit of rituximab maintenance post-autograft in patients with relapsed follicular lymphoma: 12-year follow-up of the EBMT Lymphoma Working Party Lym1 trial. Bone Marrow Transplant. 2021;56(6): 1413-1421.

39. Sarkozy C, Trneny M, Xerri L, et al. Risk factors and outcomes for patients with follicular lymphoma who had histologic transformation after response to first-line immunochemotherapy in the PRIMA trial. J Clin Oncol. 2016;34(22):2575-2582.

40. El-Najjar I, Boumendil A, Luan JJ, et al. The impact of total body irradiation on the outcome of patients with follicular lymphoma treated with autologous stem-cell transplantation in the modern era: a retrospective study of the EBMT Lymphoma Working Party. Ann Oncol. 2014:25(11):2224-2229.

41. Hoskin P, Popova B, Schofield O, et al. 4 Gy versus 24 Gy radiotherapy for follicular and marginal zone lymphoma (FoRT): long-term follow-up of a multicentre, randomised, phase 3, non-inferiority trial. Lancet Oncol 2021;22(3):332-340.

42. Illidge $\mathrm{T}, \quad$ Morschhauser $\mathrm{F}$ Radioimmunotherapy in follicular lymphoma. Best Pract Res Clin Haematol. 2011;24(2):279-293

43. Kolstad A, Illidge T, Bolstad N, et al. Phase 1/2a study of $177 \mathrm{Lu}$-lilotomab satetraxetan in relapsed/refractory indolent non-Hodgkin lymphoma. Blood Adv. 2020;4(17):4091.

44. Phillips TJ, Michot JM, Ribrag V. Can nextgeneration PI3K inhibitors unlock the full potential of the class in patients with B-cell lymphoma? Clin Lymphma Myeloma Leuk. 2021;21(1):8-20

45. Salles G, Schuster SJ, De Vos S, et al. Efficacy and safety of idelalisib in patients with relapsed, rituximab- and alkylating agentrefractory follicular lymphoma: a subgroup analysis of a phase 2 study. Haematologica. 2017;102(4):e156-e159.

46. Gopal AK, Kahl BS, de Vos S, et al. PI3Kठ inhibition by idelalisib in patients with relapsed indolent lymphoma. N Engl J Med. 2014;370(11):1008-1018.

47. Flinn IW, Miller CB, Ardeshna KM, et al. DYNAMO: a phase II study of duvelisib (IPI-145) in patients with refractory indolent non-Hodgkin lymphoma. J Clin Oncol. 2019;37(11):912-922.

48. Fowler NH, Samaniego F, Jurczak W, et al. Umbralisib, a dual PI3K $\delta / C K 1 \varepsilon$ inhibitor in patients with relapsed or refractory indolent lymphoma. J Clin Oncol. 2021;39(15):16091618.

49. Dreyling M, Santoro A, Mollica L, et al. Long-term safety and efficacy of the PI3K inhibitor copanlisib in patients with relapsed or refractory indolent lymphoma: 2-year follow-up of the CHRONOS-1 study. Am J Hematol. 2020;95(4):362-371.

50. Gopal AK, Kahl BS, Flowers CR, et al. Idelalisib is effective in patients with highrisk follicular lymphoma and early relapse after initial chemoimmunotherapy. Blood. 2017;129(22):3037-3039

51. Lynch R, Paneesha S, Avigdor A, et al. Phase 2 study evaluating the efficacy and safety of parsaclisib in patients with relapsed or refractory follicular lymphoma (CITADEL203). Blood. 2020;136(Suppl 1):36-37.

52. Zelenetz AD, Jagadeesh D, Reddy NM, et al. Results of the PI3K $\delta$ inhibitor ME-401 alone or with rituximab in relapsed/refractory (R/R) follicular lymphoma (FL). J Clin Oncol. 2019:37(15 suppl):7512

53. Matasar MJ, Capra M, Özcan M, et al. Copanlisib plus rituximab versus placebo 
Recent developments for patients with R/R FL

plus rituximab in patients with relapsed indolent non-Hodgkin lymphoma (CHRONOS-3): a double-blind, randemised, placebo-controlled, phase 3 trial. Lancet Oncol. 2021;22(5):678-689.

54. Copal AK, Schuster SJ, Fowler NH, et al. Ibrutinib as Treatment for patients with relapsed/refractory follicular lymphoma: results from the open-label, multicenter, phase II DAWN study. J Chin Oncol. 2018;36(23):2405-2412.

55. Mu N, Wang F, Sun T, et al. Follicular lymphoma-associated BTK mutations are Inactivating resulting in augmented AKT activation. Olin Cancer Res. 2021;27(8):2301-2313.

56. Fowler NH, Coleman M, Stevens DA, et al. Acalabrutinib alone or in combination with rituximab (R) in follicular lymphoma (FL). J Chin Oncol. 2018;36(15_suppl):7549-7549.

57. Tam CS, Quach H, Nicole A, et al. Zanubrutinib (BGB-3111) plus obinutuzum$\mathrm{ab}$ in patients with chronic lymphocytic leukemia and follicular lymphoma. Blood Adv. 2020;4(19):4802-4811.

58. Bennani NN, LaPland BR, Ansell SM, et al. Efficacy of the oral mTORC1 inhibitor everolimus in relapsed or refractory indolent lymphoma. Am J Hematol. 2017;92(5):448453.

59. Smith SM, Besien K van, Garrison T, et al. Temsirolimus has activity in non-mantle cell non-Hodgkin's lymphoma subtypes: the University of Chicago phase II consortium. J Chin Oncol. 2010;28(31):4740-4746.

60. Davids MS, Roberts AW, Kenkre VP, et al. Long-term follow-up of patients with relapsed or refractory non-Hodgkin lymphoma treated with venetoclax in a phase I, first-in-human study. Chin Cancer Res. 2021;27(17):4690-4695.

61. Zinzani PL, Fin IW, Yen SLS, et al. Venetoclax-rituximab with or without bendamustine vs bendamustine-rituximab in relapsed/refractory follicular lymphoma. Blood. 2020;136(23):2628.

62. Blombery P, Birkinshaw RW, Nguyen T, et al. Characterization of a novel venetoclax resistance mutation (BCL2 Phe104Ile) observed in follicular lymphoma. $\mathrm{Br} \mathrm{J}$ Haematol. 2019 186(6):e188-191.

63. Morschhauser F, Filly H, Chaidos A, et al. Tazemetostat for patients with relapsed or refractory follicular lymphoma: an openlabel, single-arm, multicentre, phase 2 trial. Lancet Oncol. 2020;21(11):1433-1442.

64. Ribrag V, Kim WS, Bouabdallah R, et al. Safety and efficacy of abexinostat, a pan-histone deacetylase inhibitor, in non-Hodgkin lymphoma and chronic lymphocytic leukemia: results of a phase II study. Haematological. 2017;102(5):903-909.

65. Kirschbaum M, Frankel P, Popplewell L, et al. Phase II study of vorinostat for treatment of relapsed or refractory indolent nonHodgkin's lymphoma and mantle cell lymphoma. J Chin Oncol. 2011;29(9):1198-1203.

66. Ogura M, Ando K, Suzuki T, et al. A multicentre phase II study of vorinostat in patients with relapsed or refractory indolent B-cell non-Hodgkin lymphoma and mantle cell lymphoma. $\mathrm{Br} \mathrm{J}$ Haematol. 2014;165(6):768-776.

67. Evens AM, Balasubramanian S, Vise JM, et al. A phase I/II multicenter, open-label study of the oral histone deacetylase inhibitor abexinostat in relapsed/refractory lymphoma. Chin Cancer Res. 2016;22(5):10591066.

68. Assouline SE, Nielsen TH, Mu S, et al. Phase 2 study of panobinostat with or without rit- uximab in relapsed diffuse large B-cell lymphoma. Blood. 2016;128(2):185-194.

69. Howard JA, Koniali L, D'Avola A, et al. KDM5 inhibition offers a novel therapeutic strategy for the treatment of KMT2D mutant lymphomas. Blood. 2021;138(5): 370-381.

70. Kahl BS, Hong F, Williams ME, et al. Rituximab extended schedule or retreatmont trial for low-tumor burden follicular lymphoma: Eastern Cooperative Oncology Group protocol E4402. J Chin Oncol. 2014;32(28):3096-3102.

71. Sen LH, Goy A, Offer FC, et al. Randomized phase II trial comparing obinutuzumab (GA101) with rituximab in patients with relapsed CD20+ indolent Bcell non-Hodgkin lymphoma: final analysis of the GAUSS study. J Chin Oncol. 2015;33(30):3467-3474.

72. Leonard JP, Trneny M, Izutsu K, et al. AUGMENT: a phase III study of lenalidomide plus rituximab versus placebo plus rituximab in relapsed or refractory indolent lymphoma. J Olin Oncol. 2019;37(14):11881199.

73. Davis TA, Grillo-López AJ, White CA, et al. Rituximab anti-CD20 monoclonal antibody therapy in non-Hodgkin's lymphoma: safety and efficacy of re-treatment. J Chin Oncol. 2000;18(17):3135-3143.

74. Johnston A, Bouafia-Sauvy F, BroussaisGuillaumot F, et al. Retreatment with rituximab in 178 patients with relapsed and refractory B-cell lymphomas: a single institution case control study. Leak Lymphoma. 2010;51(3):399-405.

75. Maloney DG, Ogura M, Fukuhara N, et al. A phase 3 randomized study (HOMER) of oftumumab vs rituximab in NHL relapsed after rituximab-containing therapy. Blood Adv. 2020;4(16):3886-3893.

76. Ramsay AG, Clear AJ, Kelly G, et al. Follicular lymphoma cells induce T-cell immunologic synapse dysfunction that can be repaired with lenalidomide: Implications for the tumor microenvironment and immunotherapy. Blood. 2009;114(21):47134720.

77. Chin H, Trial P, Bjorklund C, et al. Combination lenalidomide-rituximab immunotherapy activates anti-tumour immunity and induces tumour cell death by complementary mechanisms of action in follicular lymphoma. Br J Haematol. 2019;185(2):240-253.

78. Vo DN, Alexia C, Allende-Vega N, et al. NK cell activation and recovery of NK cell subsets in lymphoma patients after obinutuzumab and lenalidomide treatment. Oncoimmunology. 2018;7(4):e1409322.

79. Morschhauser F, Le Gouill S, Feugier P, et al. Obinutuzumab combined with lenalidomice for relapsed or refractory follicular Bcell lymphoma (GALEN): a multicentre, single-arm, phase 2 study. Lancet Haematol. 2019;6(8):e429-437.

80. Gosh N, Tales GA, Lassos IS, et al. Obinutuzumab (G)-atezolizumab (atezo)lenalidomide (len) for the treatment of relapsed/refractory (R/R) follicular lymphoma (FL): final analysis of a phase Ib/II trial. J Chin Oncol. 2021;39(15_suppl):7511.

81. Jurczak W, Zinzani PL, Gaidano G, et al. Phase Ila study of the CD19 antibody MOR208 in patients with relapsed or refractory B-cell non-Hodgkin's lymphoma. Ann Oncol. 2018;29(5):1266-1272.

82. Jacobson CA, Chavez JC, Sehgal AR, et al. Interim analysis of ZUMA-5: a phase II study of axicabtagene ciloleucel (axi-cel) in

patients (pts) with relapsed/refractory indolent non-Hodgkin lymphoma (R/R iNHL). J Chin Oncol. 2020;38(15_suppl):8008.

83. Jacobson CA, Chavez JC, Sehgal A, et al. Outcomes in ZUMA-5 with axicabtagene ciloleucel (axi-cel) in patients (pts) with relapsed/refractory (R/R) indolent nonHodgkin lymphoma (iNHL) who had the high-risk feature of progression within 24 months from initiation of first anti-CD20 containing chemoimmunotherapy (POD24). J Chin Oncol. 2021;39(15_suppl):7515.

84. Ghione P, Patel A, Bobillo S, Deighton K, Jacobson C, Nahas M. A comparison of chinical outcomes from ZUMA-5 (axicabtagene ciloleucel) and the international SCHOLAR5 external control cohort in relapsed/refractory follicular lymphoma (R/R FL). European Hematology Association Congress. 2021.

85. Fowler NH, Dickinson M, Dreyling M, et al. Efficacy and safety of tisagenlecleucel in adult patients with relapsed/refractory follicular lymphoma: interim analysis of the phase 2 Elara trial. Blood. 2020;136(Suppl 1):1-3.

86. Qualls D, Gales GA. Optimizing CAR T cell therapy in lymphoma. Hematol Oncol. 2021;39 (Suppl 1):104-112.

87. Sen LH, Kamdar M, Herrera AF, et al. Randomized phase 2 trial of polatuzumab vedotin (pola) with bendamustine and rituxmab (BR) in relapsed/refractory (r/r) FL and DLBCL. J Chin Oncol. 2018;36(15_suppl): 7507.

88. Caimi PF, Ai W, Alderuccio JP, et al. Loncastuximab tesirine in relapsed or refractory diffuse large B-cell lymphoma (LOTIS2): a multicentre, open-label, single-arm, phase 2 trial. Lancet Oncol. 2021;22(6):790800 .

89. Morschhauser F, Fin IW, Advani R, et al. Polatuzumab vedotin or pinatuzumab vedotin plus rituximab in patients with relapsed or refractory non-Hodgkin lymphoma: final results from a phase 2 randomised study (ROMULUS). Lancet Haematol. 2019;6(5):e254-265.

90. Diefenbach C, Kohl BS, Banerjee L, et al. Polatuzumab vedotin plus obinutuzumab and lenalidomide in patients with relapsed/refractory follicular lymphoma: pimary analysis of the full efficacy population in a phase Ib/II trial. Blood. 2019;134 (Suppl_1):126

91. Hamadan M, Redford J, Carlo-Stella C, et al. Final results of a phase 1 study of loncastuximab tesirine in relapsed/refractory B-cell non-Hodgkin lymphoma. Blood. 2021;137 (19):2634-2645.

92. Assouline SE, Kim WS, Sen LH, et al. Mosunetuzumab shows promising efficacy in patients with multiply relapsed follicular lymphoma: updated clinical experience from a phase I dose-escalation trial. Blood. 2020;136(Suppl 1):42-44.

93. Hutchings $M$, Morschhauser F, Iacoboni G, et al. Glofitamab, a novel, bivalent CD20targeting $\mathrm{T}$-cell-engaging bispecific antibody, induces durable complete remissions in relapsed or refractory B-cell lymphoma: a phase I trial. J Chin Oncol. 2021;39(18):19591970.

94. Hutchings M, Mouse R, Clause MR, et al. Dose escalation of subcutaneous epcoritam$\mathrm{ab}$ in patients with relapsed or refractory Bcell non-Hodgkin lymphoma: an open-label, phase 1/2 study. Lancet. 2021;6736(21):1-13. 95. Bannerji R, Allan JN, Arnason JE, et al. Odronextamab (REGN1979), a human CD20 x CD3 bispecific antibody, induces durable, complete responses in patients with 
highly refractory B-cell non-Hodgkin lymphoma, including patients refractory to CAR T therapy. Blood. 2020;136(Suppl 1):42-43.

96. Feng $M$, Jiang W, Kim BYS, Zhang CC, Fu YX, Weissman IL. Phagocytosis checkpoints as new targets for cancer immunotherapy. Nat Rev Cancer. 2019:19(10):568-586.

97. Advani R, Bartlett NL, Smith SM, et al. The first-in-class anti-CD47 antibody HU5F9-G4 + rituximab induces durable responses in relapsed/refractory DLBCL and indolent lymphoma: interim phase $1 \mathrm{~B} / 2$ results. Hematol Oncol. 2019;37(S2):89-90.

98. Kim TM, Lakhani N, Gainor J, et al. ALX148, a CD47 blocker, in combination with rituximab in patients with nonHodgkin lymphoma. Blood. 2020;136(Suppl 1):13-14.

99. Patel K, Ramchandren $R$, Maris $M$, et al. Investigational CD47-blocker TTI-622 shows single-agent activity in patients with advanced relapsed or refractory lymphoma: update from the ongoing first-in-human dose escalation study. Blood. 2020;136 (Suppl 1):46-47.

100. Dheilly E, Moine V, Broyer L, et al. Selective blockade of the ubiquitous checkpoint receptor CD47 is enabled by dual-targeting bispecific antibodies. Mol Ther. 2017;25(2): 523-533.

101. Nakamura K, Casey M, Oey $\mathrm{H}$, et al. Targeting an adenosine-mediated "don't eat me signal" augments anti-lymphoma immunity by anti-CD20 monoclonal antibody. Leukemia. 2020;34(10):2708-2721.

102. Liu X, Pu Y, Cron K, et al. CD47 blockade triggers $\mathrm{T}$ cell-mediated destruction of immunogenic tumors. Nat Med. 201521(10):
1209-1215.

103.Laurent C, Charmpi K, Gravelle P, et al Several immune escape patterns in nonHodgkin's lymphomas. Oncoimmunology. 2015;4(8):e1026530

104. Armand P, Janssens A, Gritti G, et al Efficacy and safety results from CheckMate 140 , a phase 2 study of nivolumab for relapsed/refractory follicular lymphoma. Blood. 2021;137(5):637-645.

105. Ding W, Laplant B, Witzig TE, et al. PD-1 blockade with pembrolizumab in relapsed low grade non-Hodgkin lymphoma. Blood. 2017; 130(Suppl 1):4055.

106. Salles G, Ghosh N, Lossos IS, et al. Atezolizumab in combination with obinutuzumab and lenalidomide demonstrates favorable activity and manageable toxicity in patients with relapsed/refractory follicular lymphoma (FL): an interim analysis of a phase Ib/II trial. Blood. 2018;132(Suppl 1): 1603.

107. Palomba ML, Till BG, Park SI, et al. A phase IB study evaluating the safety and clinical activity of atezolizumab combined with obinutuzumab in patients with relapsed or refractory non-Hodgkin lymphome (NHL). Hematol Oncol. 2017;35(S2):137-138.

108. Nastoupil LJ, Westin JR, Fowler NH, et al. Response rates with pembrolizumab in combination with rituximab in patients with relapsed follicular lymphoma: interim results of an on open-label, phase II study. J Clin Oncol. 2017;35(15_suppl):7519.

109. Houot R, Goldstein MJ, Kohrt HE, et al. Therapeutic effect of CD137 immunomodulation in lymphoma and its enhancement by Treg depletion. Blood. 2009;114(16):
3431-3438.

110. Gopal AK, Levy R, Houot R, et al. First-inhuman study of utomilumab, a 4 1BB/CD137 agonist, in combination with rituximab in patients with follicular and other CD20+ non-Hodgkin lymphomas. Clin Cancer Res. 2020;26(11):2524-2534.

111. Segal NH, Logan TF, Hodi FS, et al. Results from an integrated safety analysis of urelumab, an agonist anti-CD137 monoclonal antibody. Clin Cancer Res. 2017;23(8):19291936.

112. Timmerman J, Herbaux C, Ribrag V, et al Urelumab alone or in combination with rituximab in patients with relapsed or refractory B-cell lymphoma. Am J Hematol. 2020;95(5):510-520

113. Xu Y, Szalai AJ, Zhou T, et al. FcyRs modulate cytotoxicity of anti-Fas antibodies: implications for agonistic antibody-based therapeutics. J Immunol. 2003;171(2):562 568.

114. Herter S, Sam J, Ferrara Koller C, et al. RG6076 (CD19-4-1BBL): CD19-targeted 41BB ligand combination with glofitamab as an off-the-shelf, enhanced T-cell redirection therapy for B-cell malignancies. Blood. 2020;136(Suppl 1):40.

115. Andorsky DJ, Coleman M, Yacoub A, et al. MAGNIFY phase IIIb interim analysis of induction R2 followed by maintenance in relapsed/refractory indolent NHL. J Clin Oncol. 2020;38(15_suppl):8046.

116. Dreyling M, Santoro A, Leppä S, et al Efficacy and safety in high-risk relapsed or refractory indolent follicular lymphoma patients treated with copanlisib. Hematol Oncol. 2019;37(Suppl):387-389. 